\title{
Secondary Neutron-Production Cross Sections from Heavy-Ion Interactions between 230 and $600 \mathrm{MeV} /$ nucleon
}

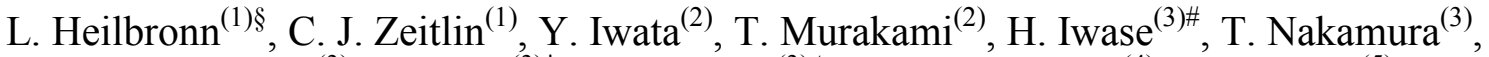

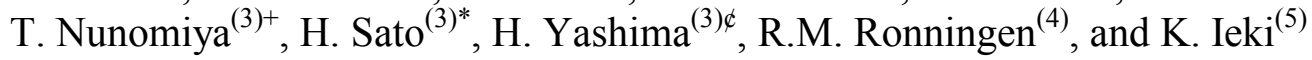

(1) MS 74-197, Lawrence Berkeley National Laboratory, Berkeley, CA, 94720

(2) Department of Accelerator Physics and Engineering, National Institute of Radiological Sciences, 4-9-1 Anagawa, Inage, Chiba 263-8555, Japan

(3) Cyclotron and Radioisotope Center, Tohoku University, Aoba, Aramaki, Sendai 9808578, Japan

(4) National Superconducting Cyclotron Laboratory, Michigan State University, East Lansing, MI 48824-1321

(5) Department of Physics, Rikkyo University, 3-34-1 Nishi Ikebukuro, Toshima, Tokyo 171-8501, Japan

\# Present address: Gesellschaft für Schwerionenforschung, Darmstadt, Germany

* Present address: Isogo Nuclear Engineering Center, Toshiba Co. 8 Shinsugita-Cho, Isogo-Ku, Yokohama 235-8523, Japan

+ Present address: Fuji Electric Systems Co., Ltd., Radiation Equipment Dept., Tokyo

Factory, 1, Fuji-machi, Hino-city, Tokyo 191-8502, Japan

$\notin$ Present address: Reactor Research Institute, Kyoto University,

$\S$ E-mail: LHHeilbronn@,LBL.gov

Contact author:

Lawrence Heilbronn

MS 74-197

Lawrence Berkeley National Laboratory

Berkeley, CA 94720

lhheilbronn@1bl.gov

fax: 510-486-6949

Number of pages (double spaced): 64

Number of figures: 30

Number of tables: 7 


\begin{abstract}
Secondary neutron-production cross-sections have been measured from interactions of $230 \mathrm{MeV} /$ nucleon $\mathrm{He}, 400 \mathrm{MeV} /$ nucleon N, $400 \mathrm{MeV} /$ nucleon $\mathrm{Kr}, 400$ $\mathrm{MeV} /$ nucleon Xe, $500 \mathrm{MeV} /$ nucleon Fe, and $600 \mathrm{MeV} /$ nucleon Ne interacting in a variety of elemental and composite targets. We report the double-differential production cross sections, angular distributions, energy spectra, and total cross sections from all systems. Neutron energies were measured using the time-of-flight technique, and were measured at laboratory angles between $5^{\circ}$ and $80^{\circ}$. The spectra exhibit behavior previously reported in other heavy-ion-induced neutron production experiments; namely, a peak at forward angles near the energy corresponding to the beam velocity, with the remaining spectra generated by preequilibrium and equilibrium processes. The doubledifferential spectra are fitted with a moving-source parameterization. Observations on the dependence of the total cross sections on target and projectile mass are discussed.
\end{abstract}




\section{INTRODUCTION}

Advancements in heavy-ion accelerators over the last 30 years have led to the development of several new areas of research, such as hadron radiotherapy for cancer patients, shielding design studies for deep space missions, and the design and construction of high-intensity radioactive beam facilities. For each of these fields, the copious production of secondary neutrons from interactions of the primary heavy-ion beam is a concern. In the case of hadron radiotherapy, as the beam transports through the body to the tumor site, nuclear interactions can produce neutrons, which in turn can lead to dose in healthy tissue outside the treatment area. In the case of shielding for missions in space, the production of neutrons from interactions of galactic cosmic rays (GCR) can make up a significant portion of the total dose received in those missions. For example, theoretical models have shown secondary neutrons to be a major contributor to exposures within lunar habitats and on the Martian surface, ${ }^{1}$ and recent studies have shown that neutrons could comprise 30 percent of the dose equivalent on the ISS. ${ }^{2}$ In the case of radioactive beam facilities, such as the Department of Energy's planned Rare Isotope Accelerator (RIA) project, ${ }^{3}$ the production of secondary neutrons will have an effect on the lifetime of key components in the accelerator, as well as have consequences in the design of the shielding for the facility.

Because of the complexity of the applied research mentioned above, much of the work in those fields involves the use of heavy-ion transport model calculations. Both deterministic and Monte Carlo approaches can be applied to transport model calculations. Both methods have inherent advantages and disadvantages, and as such the application of those methods very much depends on the particular problem being addressed. A 
deterministic approach for heavy ion transport in complex media has been developed at the NASA Langley Research Center since the mid 1980s. It has yielded a family of radiation transport codes mainly dedicated to solving engineering problems in radiationshielding analysis for space missions. ${ }^{4-6}$ One of these deterministic codes is the highcharge-and-energy transport code HZETRN, based on the one-dimensional formulation of the Boltzmann transport equation with a straight-ahead approximation ${ }^{7}$ and a semiempirical abrasion-ablation fragmentation model for nuclear fragmentation processes. Using simplifying approximations, the radiation field in and around shielding materials can be calculated by this code with an acceptable accuracy for space research and dosimetry.

Several Monte Carlo computer codes exist, or are in the stage of development, for the simulation of the transport of light and heavy ions in matter. Two codes for the simulation of hadronic cascades (neutrons, protons, pions), $\mathrm{HETC}^{8}$ and SHIELD, ${ }^{9}$ were developed in the early seventies in the US (Oak Ridge) and the former USSR (Dubna), respectively. These two codes have evolved considerably since then. Various spin-offs of HETC have proliferated under the names of HERMES, ${ }^{10}$ LAHET $^{11}$, MCNPX, ${ }^{12}$ NMTC/JAERI ${ }^{13}$ and PHITS. ${ }^{14,15}$ HETC itself has recently included heavy-ion interactions. ${ }^{16,17}$ Other major Monte Carlo transport codes currently in use that also include heavy-ion interactions (or are in the process of including heavy-ion interactions) are MARS,${ }^{18}$ MCNPX, ${ }^{12}$ FLUKA ${ }^{19-20}$ and GEANT4 ${ }^{21}$

The validation and verification of the output from transport model calculations depends on a reliable set of experimental nuclear data with which to compare. Until recently, the thin-target (cross sections) and thick-target (yields) neutron-production data 
from heavy-ion interactions that was applicable to the general problem of GCR transport was scant. A number of thick-target (stopping-target) neutron yields from high-energy ( $>100 \mathrm{MeV} /$ nucleon) heavy ion experiments have been published ${ }^{22-27}$ which can be used for direct confirmation of transport model calculations of various components of the GCR field. A reliable, calculated database of neutron-production cross sections is needed as input for transport model calculations. As such, a set of experimental cross section data is needed to verify the database of calculated input cross sections. There are some existing heavy-ion neutron-production cross section measurements relevant to GCR transport. ${ }^{28-34}$ A compilation of heavy-ion-induced secondary neutron thick-target yields and cross sections is available in a recently published handbook. ${ }^{35}$ The handbook contains the data as well as descriptions of the experiments and analyses. The data presented in this work augments other published data to provide a broad base of experimental data suitable for the study of systematics in secondary neutron production.

\section{EXPERIMENTAL DETAILS}

The measurements took place in between May of 2001 and July of 2003 at the Heavy Ion Medical Accelerator in Chiba (HIMAC) facility of the National Institute of Radiological Sciences (NIRS), Japan. Table I indicates the beam ions, beam energies and targets used in those measurements. The target thicknesses are given in units of $\mathrm{g} / \mathrm{cm}^{2}$. All targets except the Li target were $10-\mathrm{cm}$ by $10-\mathrm{cm}$ square. The Li target was a cylinder with its cylindrical axis aligned along the beam direction. The diameter of the $\mathrm{Li}$ target was $5.7 \mathrm{~cm}$.

Figure 1 shows a schematic diagram (not to scale) of the experimental arrangement used for the measurements. The placement of the neutron detectors is 
identical in angle and path length to the arrangement described in Refs. 33 and 34, however it is important to note these experiments were conducted on a different beam line at HIMAC than the one used in Refs. 33 and 34. The most important difference in the beam line used for this study is that the beam dump is much closer to the neutron detectors, which required the augmentation of the existing dump on that beam line with additional shielding materials. In Fig. 1, shielding material drawn with diagonal lines is steel (or iron), and the material drawn with the dotted fill is concrete. In order to reduce the number of beam interactions that occur in air between the target location and the beam dump, a thin-walled He-filled tube was placed along the beam axis.

As shown in Fig. 1, neutron detectors were placed between $5^{\circ}$ and $80^{\circ}$, at varying flight paths from the target position. Table II contains information regarding the positions of each detector, including the acceptance of the detector given in millisteradians. The flight path lengths indicate the distance from target center to detector center, in $\mathrm{cm}$. Each neutron detector is a cylindrical cell of liquid scintillator (NE-213), $12.7 \mathrm{~cm}$ in diameter and $12.7 \mathrm{~cm}$ long. The detectors were oriented such that the cylindrical axis was along the line connecting the target center to the detector center. Each detector cell was directly coupled to a $12.7-\mathrm{cm}$ diameter phototube. The intrinsic timing resolution of these detectors, as measured with ${ }^{60} \mathrm{Co}$ source, is on the order of 700800 ps.

A 5-mm thick, $12.7-\mathrm{cm}$ by $12.7-\mathrm{cm}$ square, solid plastic (NE-102) scintillator (referred to as "veto detector") was placed in front of each neutron detector. The veto detectors were used to tag events in the neutron detectors that came from charged particles produced in the target. A Monte Carlo calculation estimated that approximately 
$1 \%$ of the neutron flux is lost due to interactions in the veto detector. This percentage was considered low enough that neutron losses in the veto detector were ignored.

The iron shadow bars indicated in Fig. 1 were placed between the detector and target at various times during the measurements. These bars were $60-\mathrm{cm}$ long and $15 \mathrm{~cm}$ by $15 \mathrm{~cm}$ square. When placed in front of the neutron detector, they block neutrons coming directly from the target, allowing only background neutrons (such as roomscattered neutrons) to enter the detector. Because neutron energies were determined using the time-of-flight technique, the background neutrons had to be eliminated to ensure that only neutrons travelling along a known flight path were included in the final analysis. Also, the elimination of background neutrons ensures that the direction of the secondary neutron immediately after the interaction was known. A total of two shadow bars were used, and their positions were shifted from detector to detector during the measurements in order that each neutron detector had an adequate determination of its background spectrum. In general, about $1 / 4$ of the beam time was used for background determination for each detector.

One major difference in these measurements as compared with the two previously reported measurements ${ }^{33,34}$ is that data was also taken with no target in position while beam was on. The no-target runs were taken with shadow bars both in and out. By doing so, a measure of the neutron production from materials near the target (such as the exit window, trigger-plastic detector, and air gap) was obtained. That measurement is needed because when the target is in place and a shadow bar is put into position between the target and detector, the shadow bar also blocks neutrons produced from those nearby 
sources. Thus, neutrons produced by nearby sources will be misidentified as neutrons coming from the target, unless a target-out measurement is made.

Beam pulses were delivered on target every 3.3 seconds. Pulse length varied from 0.5 seconds to 1 second, depending on beam ion and energy. Typical pulse intensities varied between $10^{4}$ and $10^{5}$ particles per pulse. The beam spot size was a few millimeters in diameter, and the beam divergence was negligible compared with the spread of the beam due to Coulomb scattering through the target and air. The beam exited the vacuum beam line through a $100-\mu \mathrm{m}$-thick aluminum window and passed through either a $0.5-\mathrm{mm}$-thick or $0.1-\mathrm{mm}$-thick, 30-cm diameter NE102 scintillator. The scintillator was placed approximately $5 \mathrm{~cm}$ downstream from the exit window. That scintillator, referred to as the trigger detector, was used to count the number of beam particles incident upon the target. It was also used to provide a timing signal for a timeof-flight measurement with each event in the neutron detectors. After passing through the trigger detector, the beam then passed through the target position, approximately 19$\mathrm{cm}$ downstream from the exit window. The beam then passed through a 4-meter long, 30-cm diameter He-filled tube and stopped in a beam dump located approximately 7 meters downstream from the target position.

\section{ANALYSIS}

\section{III.A. Data Reduction, Corrections, and Normalization}

The analysis of the data presented in this paper is the same as the analyses reported in Refs. 33 and 34, except in this case there is an additional subtraction of the target-out measurement (see section above). The main points of the analysis will be described here. Detailed descriptions may be found in Refs. 33 and 34. 
The data were acquired on an event-by-event basis. Charged particle events in the neutron detectors were separated from other events when the accompanying veto detector registered a pulse height above threshold. Gamma-ray events were separated from neutron events using the pulse-shape-discrimination properties of the NE-213 cells. Neutron energies were determined using a time-of-flight technique where the signal from the neutron detector was used as the timing start, and the signal from the trigger plastic was used as the stop. An absolute time scale was determined by locating the prompt gamma-ray peak in the raw time-of-flight spectra. The overall timing resolution, as measured by the width of the prompt gamma-ray peak, was on the order of $1 \mathrm{nsec}$ for each detector. Where possible, the data were corrected for excessive constant-fractiondiscriminator (CFD) walk using an off-line analysis technique. ${ }^{36}$ The minimum time-todigital (TDC) bin-width was set to $1 \mathrm{~ns}$ in the offline analysis. Where needed, bin widths were increased to reduce the statistical uncertainty. In general, the statistical uncertainties were kept to $20 \%$ or less, although in some cases the statistical uncertainties were higher (up to $50 \%$ for some points in the double differential spectra).

The spectra were normalized to the number of incoming beam ions, as measured by the trigger detector. The number of neutron events in a particular energy bin was corrected for the energy-dependent detection efficiency. In general, detection efficiencies ranged between 10 and 40 percent above the pulse height threshold. Pulse-height energy calibration was done for each detector using gamma ray sources and the proton-recoil calibration method. ${ }^{26}$

The spectra were corrected for neutron flux attenuation due to transport through the target and air gap between the target and neutron detector. For the purposes of 
transport calculations, it may be preferred to have the data without corrections for neutron flux attenuation. If so desired, the uncorrected data is available in Ref. 35. The amount of attenuation was calculated using a Monte Carlo code that incorporates relevant elastic and non-elastic neutron-scattering cross sections. Some of the neutron-interaction crosssection data bases extend to neutron energies of $1000 \mathrm{MeV}$; however, most of the data bases used in the code only report cross sections for neutron energies up to $150 \mathrm{MeV}$. For those cases, cross sections above $150 \mathrm{MeV}$ were assumed to be equal to the value at $150 \mathrm{MeV}$. For purposes of the calculation, interactions that produce neutrons were assumed to occur at the midpoint of the target. From that point, neutrons were transported through the remaining target and air gap, and neutrons that did not make it to the neutron detector were tallied. Figure 2 shows the percentage of transmitted neutron flux as a function of neutron energy, angle, and target material. Neutron flux attenuation was greatest for the $230 \mathrm{MeV} /$ nucleon He systems, leading to an approximate $15 \%$ correction in the total cross section. For other systems with heavier projectiles and thinner targets than the $230 \mathrm{MeV} /$ nucleon He systems, the correction to the total cross sections was as little as 3 to 5 percent. The amount of attenuation is greatest at $80^{\circ}$ for all beams and targets.

\section{III.B. Systematic Uncertainties}

The systematic uncertainties considered here include estimated and measured effects in: (1) correction for detection efficiency, (2) correction for loss of neutron flux, (3) uncertainty in the solid angle subtended by each detector, (4) uncertainty in the calibration of the charge-to-digital (QDC) data, referred to as "pulse-height calibration" above, and (5) the uncertainty in the number of beam particles counted by the beam 
scintillator (trigger detector). A detailed discussion of the how the uncertainties were determined for the first four items may be found in Ref 34 . The uncertainty in solid angle may be found in Table II. The uncertainty in the number of beam particles was determined by estimating the number of events that had two beam particles striking the trigger detector close enough in time that they only generated one CFD pulse that was counted. This was determined by looking at the QDC spectrum for the trigger detector, and estimating the number of events that had a total integrated charge that was appreciably greater than the total integrated charge from just one beam particle. The fraction of events that had more than one beam particle depended on beam conditions, but in the worst case it was no greater than four percent of the events. It was conservatively assumed that each beam had an uncertainty of four percent in the number of beam particles.

Table III shows the percent uncertainty for all five items as a function of detector number (re: Table II). The overall systematic uncertainty (column 7 of Table III) was determined by adding the individual uncertainties in quadrature. The systematic uncertainties were applied to the angular distributions and total cross sections.

\section{DOUBLE-DIFFERENTIAL SPECTRA}

Figures 3 through 24 show the double differential spectra from all the systems identified in Table I. The error bars represent the statistical uncertainties in the data. The solid lines indicate fits to the data using a moving source parameterization (see discussion below). The spectra shown in Figs. 3 through 24 follow the same general trends seen in similar data. ${ }^{32-34}$ The spectra at $5^{\circ}$ are dominated by neutrons from the breakup of the 
projectile, resulting in a peak that is centered near the beam ion's specific energy (the beam's energy in units of energy-per-nucleon). At $10^{\circ}$ and $20^{\circ}$, evidence of neutrons from projectile-like fragments can still be seen, although the peaks are broader and centered at lower energies. The peak or bump at $20^{\circ}$ due to projectile fragmentation is most clearly seen for the lighter mass projectiles. At larger angles, the spectra appear to be generated from two distinct sources: (1) Evaporation from the target residues that dominates the spectra below $20 \mathrm{MeV}$, and (2) decay of the overlap region between the projectile and target that produce neutrons with energies from a few $\mathrm{MeV}$ up to hundreds of $\mathrm{MeV}$.

The solid lines in Figs. 3 through 24 show fits to the data using a moving source parameterization. Three sources were assumed in the fitting: (1) breakup of the projectile, (2) breakup of the decay of the overlap region, referred to as pre-equilibrium emission, and (3) decay of the target remnant.

As was done in Ref.33, the projectile-like source was assumed to have the form

$\frac{d^{2} \sigma}{p_{c}^{2} d p_{c} d \Omega_{c}}=N \exp \left(-\frac{p_{c}^{2}}{2 \sigma^{2}}\right)$,

where $\mathrm{p}_{\mathrm{c}}$ is the momentum of the neutron in the rest frame of the source and $\sigma$ is a width parameter that is related to the internal momentum of nucleons within the source. ${ }^{37}$ The double-differential spectra reported here are related to the cross section in Eqn. 1 by

$\frac{d^{2} \sigma}{d E d \Omega}=p E_{c} \frac{d^{2} \sigma}{p_{c}^{2} d p_{c} d \Omega_{c}}$,

where $E_{c}$ is the neutron's kinetic energy in the source's frame, and $p$ is the neutron's momentum in the lab frame. $\mathrm{E}_{\mathrm{c}}$ is related to the kinetic energy in the lab frame by 
$E_{c}=\gamma(E-\beta p \cos \theta)$,

where $\beta$ is the source velocity $(\mathrm{v} / \mathrm{c}), \gamma$ is the Lorentz factor, and $\theta$ is the lab angle.

The other two sources assume an isotropic decay in their rest frame and are given the following Maxwellian form (in the rest frame) as:

$\frac{d^{2} \sigma}{p_{c}^{2} d p_{c} d \Omega_{c}}=\frac{N}{(2 \pi T)^{3 / 2}} \exp \left(-\frac{p_{c}^{2}}{2 m T}\right)$.

Table IV shows the fit parameters for all systems except the $500 \mathrm{MeV} /$ nucleon Fe

$+\mathrm{CH}_{2}$ system. The first part of the table shows the parameters from Eqn. 1, and the second half shows the parameters from the two Maxwellian sources described in Eqn. 4. Because there was no data at $5^{\circ}$ and $10^{\circ}$ for the $500 \mathrm{MeV} /$ nucleon Fe systems, the parameters from Eqn. 1 were difficult to extract for those two systems. In general, the fits are adequate in describing the magnitudes and shape of the spectra between $5^{\circ}$ and $80^{\circ}$. Because the moving-source parameterization does not yield unique solutions, these fits are unsuitable for extrapolating to larger angles, or to systems with beam or target masses much different than the ones described here.

\section{ANGULAR DISTRIBUTIONS}


Figures $25-29$ show the angular distribution spectra for the indicated systems. The data points, shown with open and closed symbols, were obtained by integrating the experimental double-differential spectra over energy for neutron energies greater than 10 $\mathrm{MeV}$. The error bars include the statistical and systematic uncertainties added together in quadrature. The lines show fits to the data using the following parameterization:

$d \sigma / d \Omega=a_{1} \exp \left(-a_{2} \theta\right)+a_{3} \exp \left(-a_{4} \theta\right)$,

where $\theta$ is in radians, and $a_{1}, a_{2}, a_{3}$, and $a_{4}$ are the fit parameters. Table $\mathrm{V}$ shows the

fitted parameters from all systems. The first two terms in Eqn. 5 represent the breakup of the projectile, and the last two terms represent the contribution from the decay of the overlap region and the target remnant. Because no data were taken at $5^{\circ}$ and $10^{\circ}$ for the $500 \mathrm{MeV} /$ nucleon Fe systems, no fits were made to their angular distributions. The projectile decay parameters for the $400 \mathrm{MeV} /$ nucleon $\mathrm{Kr}+\mathrm{Pb}$ system and the 400 $\mathrm{MeV} /$ nucleon $\mathrm{N}+\mathrm{C}$ system are anomalous due to fitting the relatively low yield at $10^{\circ}$ (see Figs. 25 and 26). All of the systems exhibit a strong focussing of the spectra in the forward direction. Because a neutron energy threshold of $10 \mathrm{MeV}$ was used, a sizable fraction of the yield from the decay of the target remnant is missing, which in effect enhances the forward-focussing of the angular distributions.

\section{TOTAL CROSS SECTIONS}

The angular distribution data shown in figs. $25-29$ were integrated over angle to yield the total cross sections. The angular cross sections at $5^{\circ}$ were integrated over the range of $0^{\circ}$ to $7.5^{\circ}$, the $10^{\circ}$ yields were integrated from $7.5^{\circ}$ to $15^{\circ}$, the $20^{\circ}$ yields were integrated from $15^{\circ}$ to $25^{\circ}$, the $30^{\circ}$ yields were integrated from $25^{\circ}$ to $35^{\circ}$, the $40^{\circ}$ yields 
were integrated from $35^{\circ}$ to $50^{\circ}$, the $60^{\circ}$ yields were integrated from $50^{\circ}$ to $70^{\circ}$, and the $80^{\circ}$ yields were integrated from $70^{\circ}$ to $90^{\circ}$. Table VI shows those results for neutron energy thresholds of $5 \mathrm{MeV}$ (column three) and $10 \mathrm{MeV}$ (columns 4-6), and for yields with (columns 3, 4, and 6) and without (column 5) corrections for neutron attenuation. The total cross sections from $0^{\circ}$ to $180^{\circ}$ were deduced by adding the data-integrated cross sections from $0^{\circ}$ to $90^{\circ}$ together with the calculated total cross sections from $90^{\circ}$ to $180^{\circ}$. The total cross sections from $90^{\circ}$ to $180^{\circ}$ were calculated by integrating Eqn. 5 from $90^{\circ}$ to $180^{\circ}$, using the parameters listed in Table V. Comparing columns 4 and 5 in Table VI, it is seen that approximately 3 to 8 percent of the total cross section is estimated to be lost due to neutron flux attenuation, at least for the thinner targets used. For the thicker targets used with the $230 \mathrm{MeV} /$ nucleon He beams, about $15 \%$ of the flux is estimated to be lost.

Using the total cross sections from $0^{\circ}$ to $180^{\circ}$ in this work together with the total cross sections reported in Ref. [Iwata01], it is found that the total cross sections can be estimated using a simple parameterization that is based on the geometric cross section and the number of projectile and target neutrons from each system. The parameterization has the form:

$\sigma_{\text {neut }}($ tot $)=C \times\left(A_{\text {proj }}^{1 / 3}+A_{t \arg }^{1 / 3}\right)^{2} \times\left[\left(f_{p} \times N_{p}\right)+\left(f_{t} \times N_{t}\right)\right]$,

where $\sigma_{\text {neut }}($ tot $)$ is the total secondary-neutron-production cross section from $0^{\circ}$ to $180^{\circ}, \mathrm{C}$ is a normalization constant, $\mathrm{A}_{\text {proj }}$ and $\mathrm{A}_{\operatorname{targ}}$ are the atomic numbers of the projectile and target, $N_{p}$ and $N_{t}$ and the neutron numbers of the projectile and target, and $f_{p}$ and $f_{t}$ are the fraction of projectile and target neutrons contributing to the yield. A global fit to all of 
the data using a single set of parameters $\left(\mathrm{C}, \mathrm{f}_{\mathrm{p}}\right.$ and $\left.\mathrm{f}_{\mathrm{t}}\right)$ was somewhat successful, yielding a $\chi^{2}$ of 1.89 per degree of freedom. However, it was found that better results were obtained when the data was split into three categories: (1) light-mass projectile systems (He, C, and $\mathrm{N}$ beams), (2) intermediate-mass systems (Ne, Si, and Ar beams), and (3) heavy-mass systems ( $\mathrm{Kr}$ and $\mathrm{Xe}$ beams). Table VII contains the fit parameters from all three categories, along with the fit parameters from the global fit. The last column shows the chi-square per degree of freedom for the corresponding fits; the fits in the individual categories each yield a $\chi^{2}$ of less than 1 per degree of freedom.

Figure 30 shows the total cross sections from column 6 in Table VI and the data from Table IV in Ref. 33 extended to 180 degrees, along with the fits to the data using Eqn. 6 and the parameters from Table VII. The data for $\mathrm{C}, \mathrm{N}$, and $\mathrm{O}$ projectiles are shown with the open circles, and the fit to that set of data only (row 2 in Table VI) is shown with the solid line. The data for $\mathrm{Ne}, \mathrm{Si}$, and Ar projectiles are shown with the open triangles, and the fit to that set of data only (row 3 in Table VII) is shown with the small-dashed line. The data for heavy projectiles $(\mathrm{Kr}$ and $\mathrm{Xe})$ are shown with the filled diamond-shaped symbols, and the fit to that data set only (row 4 in Table VII) is shown with the large-dashed line. The global fit to all the data (row 5 in Table VII) is shown with the red dot-dashed line.

The trend of the parameters in Table VII as a function of projectile mass (rows 24) suggest that as the projectile mass increases, the percentage of target neutrons contributing to the yield also increases. This is consistent with the picture that, averaging over impact parameter, the larger the projectile, the larger the overlap region between target and projectile, and hence a larger fraction of target neutrons participating in the 
interaction. The parameters also suggest that as the projectile mass increases, the fraction of projectile neutrons contributing to the neutron yield decreases. This is also consistent with the interaction dynamics described above. Observation of the data in Fig. 30 suggests that the light-mass projectile data (in particular, the $\mathrm{C}+\mathrm{Pb}$ systems) forces the global fit to underestimate the data from heavier mass projectiles.

\section{Conclusions}

Neutron production cross sections, angular distributions and total cross sections were measured in a variety of heavy-ion interactions. Projectile masses ranged from $\mathrm{He}$ to $\mathrm{Xe}$, and beam energies ranged between 230 and $600 \mathrm{MeV}$ /nucleon. Targets varied in mass between $\mathrm{Li}$ and $\mathrm{Pb}$, and cross sections from a polyethylene target were measured as well. Neutrons were measured between 5 and 80 degrees, and the reported neutron energy thresholds varied between 3 and $10 \mathrm{MeV}$, depending on angle and system. A moving-source parameterization of the double-differential cross sections was performed, along with a parameterization of the angular distributions. A simple parameterization utilizing just the geometric cross section and neutron number of the target and projectile was performed on the total cross sections above $10 \mathrm{MeV}$. The systematics inferred from the parameterizations are consistent with the picture of neutron production in heavy-ions collisions: projectile breakup contributing to forward-focussed high energy yield, target evaporation contributing to the low-energy (10-20 MeV and below) yield, and decay of the overlap region contributing to a wide range of neutron energies at a wide range of angles. These data, along with other referenced data, will provide a comprehensive data base of neutron production from medium-energy heavy-ion interactions that will be useful to several transport models that are either working on the inclusion of heavy-ion 
interactions, or have just recently made public versions of the codes that do include heavy-ion interactions.

\section{ACKNOWLEDGEMENTS}

This research was supported by in part by the U.S. Department of Energy under Contract No. DEAC03076SF00098, the National Aeronautics and Space Administration under NASA Grant Nos. L14230C and H29456D, NASA Award No. H34854D, by the National Science Foundation through grant PHY-0110253, and by the Japanese Society for the Promotion of Science (JSPS) under grant ID number US02011. The experimental program was carried out as part of a research project with heavy ions at NIRS-HIMAC. The authors wish to thank Michiya Sasaki, Taishi Shiomi, and Yuuichi Tozawa for their help during the experiments. The authors also wish to thank the staff of HIMAC for their invaluable assistance during the experiment.

\section{REFERENCES}

1. L.C. SIMONSEN and J.E. NEALY, "Radiation Protection for Human Missions to the Moon and Mars," Technical Paper 3079, National Aeronautics and Space Administration (1991).

2. E.V. BENTON and G.D. BADHWAR (eds), "Prediction and Measurements of Secondary Neutrons in Space," Radiat. Meas. 33 (2001).

3. "ISOL Task Force Report to NSAC”, H. GRUNDER (chair), November 22, 1999 (unpublished).

4. J. W. WILSON, L. W. TOWNSEND, H. B. BIDASARIA, W. SCHIMMERLING, M. WONG and J. HOWARD, "Neon-20 depth-dose relations in water", Health Physics 46 No 5 (May) 1101-1111 (1984).

5. J. W. WILSON, M. REGINATO, F. HAJNAL and S. Y. CHUN, "Calculation of Dose, Dose Equivalent, and Relative Biological Effectiveness for high Charge and Energy Ions Beams", Health Physics 68 532-538 (1995). 
6. J. W. WILSON, R. K. TRIPATHI, G. D. QUALLS, F. A. CUCINOTTA, R. E. PRAEL, J. W. NORBURY, J. H. HEINBOCKEL, J. TWEED and G. De ANGELIS, "Advances in Space Radiation Shielding Codes", Journal of Radiation Research 43:Suppl. S87-S91 (2002).

7. J. W. WILSON, F. F. BADAVI, F. A. CUCINOTTA, J. L. SHINN, G. D. BADHWAR, R. SILBERGER, C. H. TSAO, L. W. TOWNSEND and R. K. TRIPATHI, "HZETRN: Description of a Free-Space Ion and Nucleon transport and Shielding Computer Program”, NASA Technical Paper 3495 (1995).

8. T. W. ARMSTRONG and K. G. CHANDLER, "HETC - A High Energy Transport Code", Nucl.Sci.Eng. 49 110-111 (1972).

9. N. M. SOBOLEVSKY, "A code for the simulation of nucleon-meson cascades in matter by the Monte Carlo method", Report JINR B1-2-5458 Dubna 1-143 (in Russian) (1970).

10. P. CLOTH, D. FILGES, R. D. NEEF, G. STERZENBACH, Ch. REUL, T. W. ARMSTRONG, B. L. COLBORN, B. ANDERS and H. BRÜCKMANN, "HERMES: A Monte Carlo System for Beam Materials Interaction Studies", KFA-Report Jül-2203 (1988).

11. R. E. PRAEL and H. LICHTENSTEIN, "User Guide to LCS: The LAHET Code System”, Los Alamos National Laboratory LA-UR-89-3014 (1989).

12. G. HUGHES, K. J. ADAMS, M. B. CHADWICK, J. C. COMLY, S. C. FRANKLE, J. S. HENDRICKS, R. C. LITTLE, R. E. PRAEL, L. S. WATERS, P. G. YOUNG Jr, "MCNPX ${ }^{\mathrm{TM}}$ - The LAHET ${ }^{\mathrm{TM}} / \mathrm{MCNP}^{\mathrm{TM}}$ Code Merger", Proc of Third Workshop on Simulating Accelerator Radiation Environments (SARE 3) KEK, Tsukuba, Japan, May 7 9 KEK Proceedings 97-5, H/R/D, Ed. H.Hirayama, 44-51 (1997).

13. H. TAKADA, N. YOSHIZAWA, K. KOSAKA, K. ISHIBASHI, “An upgraded version of the nucleon-meson transport code NMTC-JAERI97”, JAERI-Data/Code 98005 (1998).

14. H. IWASE, K. NIITA, and T. NAKAMURA, "Development of General-Purpose Particle and Heavy Ion Transport Monte Carlo", Code Journal of Nuclear Science and Technology 39 No. 11 1142-1151 (2002).

15. K. NIITA, T. SATO, H. IWASE, H. NOSE, H. NAKASHIMA, L. SIHVER, "Particle and Heavy Ion Transport Code System; PHITS", Proc. 2003 Symposium on Nuclear Data, Nov. 2003, JAERI, Takai, Japan, JAERI-Conf2004-005, pp. 33 (2004).

16. T. M. MILLER and L. W. TOWNSEND, "Comprehensive Cross Section Database Development for Generalized Three Dimensional Radiation Transport Codes”, Nucl. Sci. and Eng., Vol. 149, No. 1, January 2005, pp. 65-73. 
17. L. W. TOWNSEND, T. M. MILLER and T. A. GABRIEL, "HETC Radiation Transport Code Development for Cosmic Ray Shielding Applications in Space", Radiation Protection Dosimetry (2005, in press).

18. N. V. MOKHOV, “The MARS Code System User's Guide”, Fermilab-FN-628 (1995).

19. A. FASSÒ, A. FERRARI, J. RANFT, P. R. SALA, "New Developments in FLUKA Modeling Hadronic and EM Interactions", Proc. of 3rd Workshop on Simulating Accelerator Radiation Environments (SARE 3) KEK, Tsukuba, Japan, May 7-9, KEK Proceedings 97-5, H/R/D, Ed. H.Hirayama (1997).

20. A. FERRARI and P. R. SALA, "Intermediate and High Energy Models in FLUKA: Improvements, Benchmarks and Applications", International Conference on Nuclear Data for Science and technology, Miramare-Trieste, Italy, May 19-24, ed. By G. Reffo, A. Ventura and C. Grandi, 247-253 (1997).

21. S. AGOSTINELLI et al., GEANT4 Collaboration, "GEANT4 - a simulation tookit", Nucl. Instrum. Methods A 506, 250-303 (2003).

22. R.A. CECIL, B.D. ANDERSON, A.R. BALDWIN, R. MADEY, A. GALONSKY, P. MILLER, L. YOUNG, and F.M. WATERMAN, "Neutron Angular and Energy Distributions from 710-MeV Alphas Stopping in Water, Carbon, Steel, and Lead, and 640-MeV Alphas Stopping in Lead," Phys. Rev. C 21, 2471 (1980).

23. T. KUROSAWA, N. NAKAO, T. NAKAMURA, Y. UWAMINO, T. SHIBATA, N. NAKANISHI, A. FUKUMURA, and K. MURAKAMI, "Measurements of secondary neutrons produced from thick targets bombarded by high energy helium and carbon ions," Nucl. Sci. and Eng. 132, 30-57 (1999).

24. T. KUROSAWA, N. NAKAO, T. NAKAMURA, Y. UWAMINO, T. SHIBATA, A. FUKUMURA, and K. MURAKAMI, "Measurements of Secondary Neutrons Produced from Thick Targets Bombarded by High Energy Neon Ions," Journ. of Nucl. Sci. and Tech. 36, 41-53 (1999).

25. T. KUROSAWA, N. NAKAO, T. NAKAMURA, H. IWASE, H. SATO, Y. UWAMINO, and A. FUKUMURA, "Neutron yields from thick $\mathrm{C}, \mathrm{Al}, \mathrm{Cu}$, and $\mathrm{Pb}$ targets bombarded by $400 \mathrm{MeV} /$ nucleon Ar, Fe, Xe and $800 \mathrm{MeV} /$ nucleon Si ions," Phys. Rev. C 62, (2000).

26. L. HEILBRONN, R.S. CARY, M. CRONQVIST, F. DEAK, K. FRANKEL, A. Galonsky, K. HOLABIRD, A. HORVATH, A. KISS, J. KRUSE, R.M. RONNINGEN, H. SCHELIN, Z. SERES, C.E. Stronach, J. WANG, P. ZECHER, and C. ZEITLIN, "Neutron Yields form $155 \mathrm{MeV} /$ Nucleon Carbon and Helium Stopping in Aluminum," Nucl. Sci. and Eng. 132 (1999). 
27. B.I. BRITVICH, A.A. CHUMAKOV, R.M. RONNINGEN, R.A. BLUE, and L.H. HEILBRONN, "Measurements of thick target neutron yields and shielding studies using beams of ${ }^{4} \mathrm{He},{ }^{12} \mathrm{C}$, and ${ }^{16} \mathrm{O}$ at $155 \mathrm{MeV} /$ nucleon from the $\mathrm{K} 1200$ cyclotron at the National Superconducting Cyclotron Laboratory," Rev. Sci. Instrum. 70, 2314-2324 (1999).

28. W. SCHIMMERLING, J.W. KAST, D. ORTENDAHL, R. MADEY, R.A. CECIL, B.D. ANDERSON and A.R. BALDWIN, "Measurement of the Inclusive Neutron Production by Relativistic Neon Ions on Uranium," Phys. Rev. Lett. 43, 1985-1987 (1979).

29. R.A. CECIL, B.D. ANDERSON, A.R. BALDWIN, R. MADEY, W. SCHIMMERLING, J.W. KAST, and D. ORTENDAHL, "Inclusive neutron production by $337 \mathrm{MeV} /$ nucleon neon ions on carbon, aluminum, copper, and uranium," Phys. Rev. C 24, 2013-2029 (1981).

30. R. MADEY, B.D. ANDERSON, R.A. CECIL, P.C. TANDY, and W. SCHIMMERLING, "Total inclusive neutron cross sections and multiplicities in nucleusnucleus collisions at intermediate energies," Phys. Rev. C 28, 706-709 (1983).

31. A.R. BALDWIN, R. MADEY, W.M. ZHANG, B.D. ANDERSON, D. KEANE, J. VARGA and J.W. WATSON, "Inclusive neutron cross sections from $\mathrm{Ne}-\mathrm{Pb}$ collisions at $790 \mathrm{MeV} /$ nucleon”, Phys. Rev. C 46, 258-264 (1992).

32. H. SATO, T. KUROSAWA, H. IWASE, T. NAKAMURA, Y. UWAMINO and N. NAKAO, "Measurements of double differential neutron production cross sections by 135 MeV/nucleon He, C, Ne and $95 \mathrm{MeV} /$ nucleon Ar ions," Phys. Rev. C 64, (2001).

33. Y. IWATA, T. MURAKAMI, H. SATO, H. IWASE, T. NAKAMURA, T. KUROSAWA, L. HEILBRONN, R.M. RONNINGEN, K. IEKI, Y. TOZAWA, and K. NIITA, "Double-differential cross sections for the neutron production from heavy-ion reactions at energies E/A = 290-600 MeV," Phys. Rev. C 64, 054609 (2001).

34. L. HEILBRONN, Y. IWATA, H. IWASE, T. MURAKAMI, H. SATO, T. NAKAMURA, R.M. RONNINGEN, K. IEKI, I. GUDOWSKA, and N. SOBOLEVSKY, "Secondary neutron production cross sections from heavy ion interactions in composite targets", Phys. Rev C 73, 024603 (2006).

35. T. NAKAMURA and L. HEILBRONN, "Handbook on Secondary Particle Production and Transport by High Energy Heavy Ions", World Scientific Publishing, 5 Toh Tuck Link, Singapore 596224 (2006).

36. L. HEILBRONN, Y. IWATA, and H. IWASE, "Off-line correction for excessive constant-fraction-discriminator walk in neutron time-of-flight experiments," Nucl. Instrum. Methods A 522, 495-503 (2004). 
37. R. SERBER, "The Production of High Energy Neutrons by Stripping”, Phys. Rev. 72, 1008 (1947). 


\section{FIGURE CAPTIONS}

Fig. 1. A schematic view of the experimental arrangement used for the measurements (not to scale).

Fig. 2. The fraction of neutron flux transmitted from the target to the neutron detector as a function of neutron energy. Plots are shown for the four cases indicated in the legend.

Fig. 3. Double-differential spectra from $230 \mathrm{MeV} /$ nucleon He interacting in an $\mathrm{Al}$ target. The spectra at each laboratory angle are offset by the indicated factors of 10 . The lines come from a moving-source fit described in the text.

Fig. 4. Double-differential spectra from $230 \mathrm{MeV} /$ nucleon $\mathrm{He}$ interacting in a $\mathrm{Cu}$ target. The spectra at each laboratory angle are offset by the indicated factors of 10 . The lines come from a moving-source fit described in the text.

Fig. 5. Double-differential spectra from $400 \mathrm{MeV} /$ nucleon $\mathrm{N}$ interacting in a $\mathrm{C}$ target. The spectra at each laboratory angle are offset by the indicated factors of 10 . The lines come from a moving-source fit described in the text.

Fig. 6. Double-differential spectra from $400 \mathrm{MeV} /$ nucleon $\mathrm{N}$ interacting in a $\mathrm{Cu}$ target. The spectra at each laboratory angle are offset by the indicated factors of 10 . The lines come from a moving-source fit described in the text.

Fig. 7. Double-differential spectra from $400 \mathrm{MeV} /$ nucleon $\mathrm{Kr}$ interacting in a $\mathrm{Li}$ target. The spectra at each laboratory angle are offset by the indicated factors of 10 . The lines come from a moving-source fit described in the text. 
Fig. 8. Double-differential spectra from $400 \mathrm{MeV} /$ nucleon $\mathrm{Kr}$ interacting in a C target. The spectra at each laboratory angle are offset by the indicated factors of 10 . The lines come from a moving-source fit described in the text.

Fig. 9. Double-differential spectra from $400 \mathrm{MeV}$ /nucleon $\mathrm{Kr}$ interacting in a $\mathrm{CH}_{2}$ target. The spectra at each laboratory angle are offset by the indicated factors of 10 . The lines come from a moving-source fit described in the text.

Fig. 10. Double-differential spectra from $400 \mathrm{MeV} /$ nucleon $\mathrm{Kr}$ interacting in an $\mathrm{Al}$ target. The spectra at each laboratory angle are offset by the indicated factors of 10 . The lines come from a moving-source fit described in the text.

Fig. 11. Double-differential spectra from $400 \mathrm{MeV} /$ nucleon $\mathrm{Kr}$ interacting in a $\mathrm{Cu}$ target. The spectra at each laboratory angle are offset by the indicated factors of 10 . The lines come from a moving-source fit described in the text.

Fig. 12. Double-differential spectra from $400 \mathrm{MeV}$ /nucleon $\mathrm{Kr}$ interacting in a $\mathrm{Pb}$ target. The spectra at each laboratory angle are offset by the indicated factors of 10 . The lines come from a moving-source fit described in the text.

Fig. 13. Double-differential spectra from $400 \mathrm{MeV} /$ nucleon Xe interacting in a $\mathrm{Li}$ target. The spectra at each laboratory angle are offset by the indicated factors of 10 . The lines come from a moving-source fit described in the text.

Fig. 14. Double-differential spectra from $400 \mathrm{MeV} /$ nucleon Xe interacting in a C target. The spectra at each laboratory angle are offset by the indicated factors of 10 . The lines come from a moving-source fit described in the text. 
Fig. 15. Double-differential spectra from $400 \mathrm{MeV} /$ nucleon Xe interacting in a $\mathrm{CH}_{2}$ target. The spectra at each laboratory angle are offset by the indicated factors of 10 . The lines come from a moving-source fit described in the text.

Fig. 16. Double-differential spectra from $400 \mathrm{MeV} /$ nucleon Xe interacting in an $\mathrm{Al}$ target. The spectra at each laboratory angle are offset by the indicated factors of 10 . The lines come from a moving-source fit described in the text.

Fig. 17. Double-differential spectra from $400 \mathrm{MeV} /$ nucleon Xe interacting in a $\mathrm{Cu}$ target. The spectra at each laboratory angle are offset by the indicated factors of 10 . The lines come from a moving-source fit described in the text.

Fig. 18. Double-differential spectra from $400 \mathrm{MeV} /$ nucleon Xe interacting in a $\mathrm{Pb}$ target. The spectra at each laboratory angle are offset by the indicated factors of 10 . The lines come from a moving-source fit described in the text.

Fig. 19. Double-differential spectra from $500 \mathrm{MeV} /$ nucleon Fe interacting in a $\mathrm{Li}$ target. The spectra at each laboratory angle are offset by the indicated factors of 10 . The lines come from a moving-source fit described in the text.

Fig. 20. Double-differential spectra from $500 \mathrm{MeV} /$ nucleon Fe interacting in a $\mathrm{CH}_{2}$ target. The spectra at each laboratory angle are offset by the indicated factors of 10 .

Fig. 21. Double-differential spectra from $500 \mathrm{MeV} /$ nucleon Fe interacting in an $\mathrm{Al}$ target. The spectra at each laboratory angle are offset by the indicated factors of 10 . The lines come from a moving-source fit described in the text.

Fig. 22. Double-differential spectra from $600 \mathrm{MeV}$ /nucleon Si interacting in a $\mathrm{C}$ target. The spectra at each laboratory angle are offset by the indicated factors of 10 . The lines come from a moving-source fit described in the text. 
Fig. 23. Double-differential spectra from $600 \mathrm{MeV} /$ nucleon $\mathrm{Si}$ interacting in a $\mathrm{Cu}$ target. The spectra at each laboratory angle are offset by the indicated factors of 10 . The lines come from a moving-source fit described in the text.

Fig. 24. Double-differential spectra from $600 \mathrm{MeV}$ /nucleon Si interacting in a $\mathrm{Pb}$ target. The spectra at each laboratory angle are offset by the indicated factors of 10 . The lines come from a moving-source fit described in the text.

Fig. 25. Angular distribution from the $230 \mathrm{MeV} /$ nucleon He systems for $\mathrm{E}_{\mathrm{n}}>10$ MeV. The lines show a fit to the data using Eqn. 5.

Fig. 26. Angular distribution from the $400 \mathrm{MeV}$ /nucleon $\mathrm{N}$ systems for $\mathrm{E}_{\mathrm{n}}>10$ MeV. The lines show a fit to the data using Eqn. 5 .

Fig. 27. Angular distribution from the $400 \mathrm{MeV} /$ nucleon $\mathrm{Kr}$ systems for $\mathrm{E}_{\mathrm{n}}>10$ MeV. The lines show a fit to the data using Eqn. 5.

Fig. 28. Angular distribution from the $400 \mathrm{MeV} /$ nucleon Xe systems for $\mathrm{E}_{\mathrm{n}}>10$ MeV. The lines show a fit to the data using Eqn. 5 .

Fig. 29. Angular distribution from the $600 \mathrm{MeV} /$ nucleon Si systems for $E_{n}>10$ MeV. The lines show a fit to the data using Eqn. 5 .

Fig. 30. Total cross sections for neutrons above $10 \mathrm{MeV}$ and angles between 0 and 180 degrees, as a function of a parameter that is proportional to the geometric cross sections. The lines indicate fits to the data using Eqn. 6. 


\section{TABLE CAPTIONS}

Table I

Target species and thickness $\left(\mathrm{g} / \mathrm{cm}^{2}\right)$ used with the indicated beams

Table II

Neutron detector information. The uncertainty in the solid angle is reported as a percentage.

Table III

Systematic uncertainties as a function of detector number, expressed as a percentage.

Adding the individual uncertainties in quadrature yields the total systematic uncertainty listed in column 7.

\section{Table IV}

Parameters from moving source fits to the indicated systems. The first part of the table contains the parameters of the projectile fragmentation source, and the second part contains the parameters from the two Maxwellian sources (see Eqns. 1 and 4).

\section{Table V}

Parameters from fits to the indicated angular distributions using Eqn. 5. 


\section{Table VI}

Total cross sections for the indicated systems. Columns $3-5$ show the integrated cross sections from 0 to 90 degrees. Column six shows the deduced total cross sections from 0 to 180 degrees. 


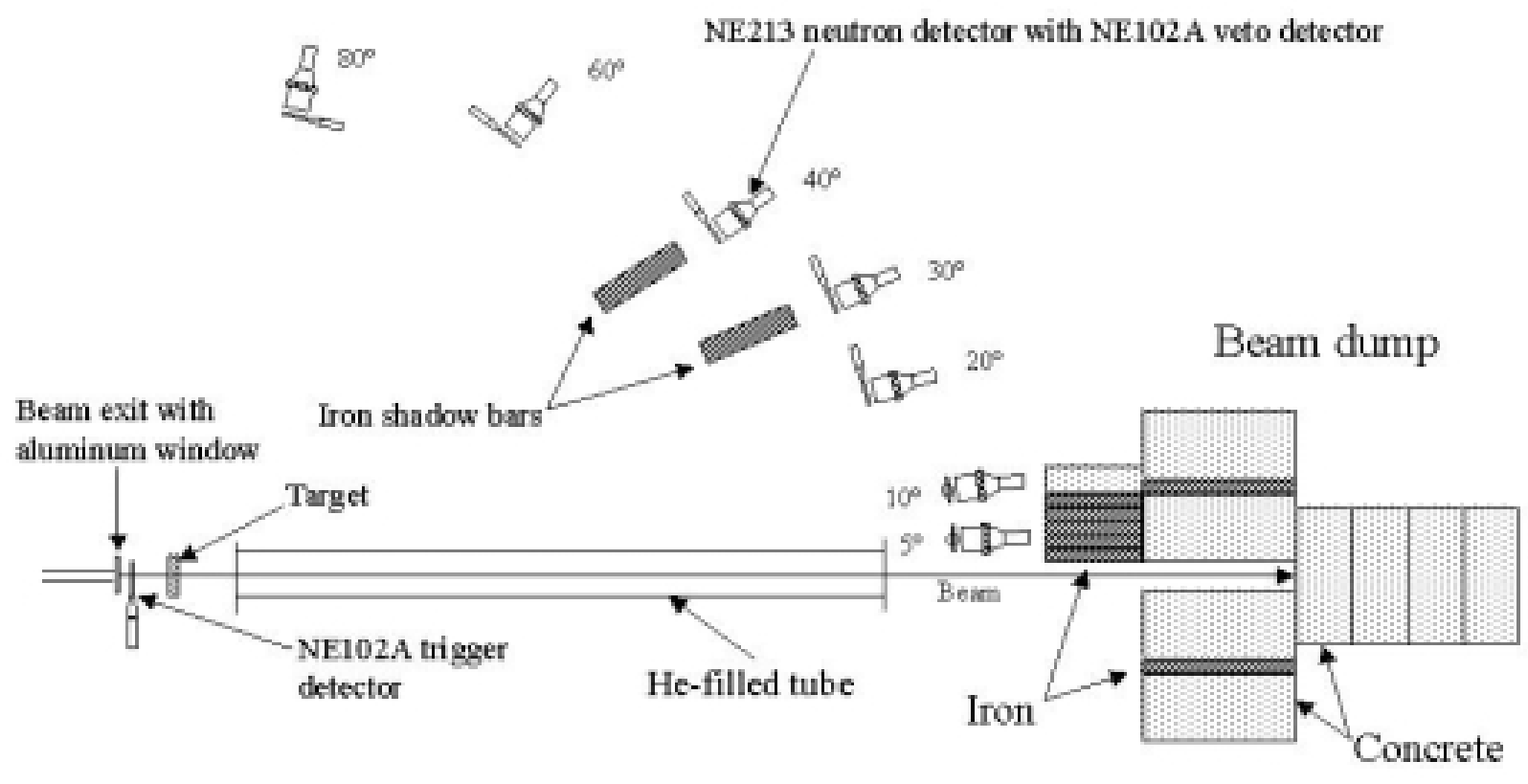

Fig. 1. A schematic view of the experimental arrangement used for the measurements (not to scale). 


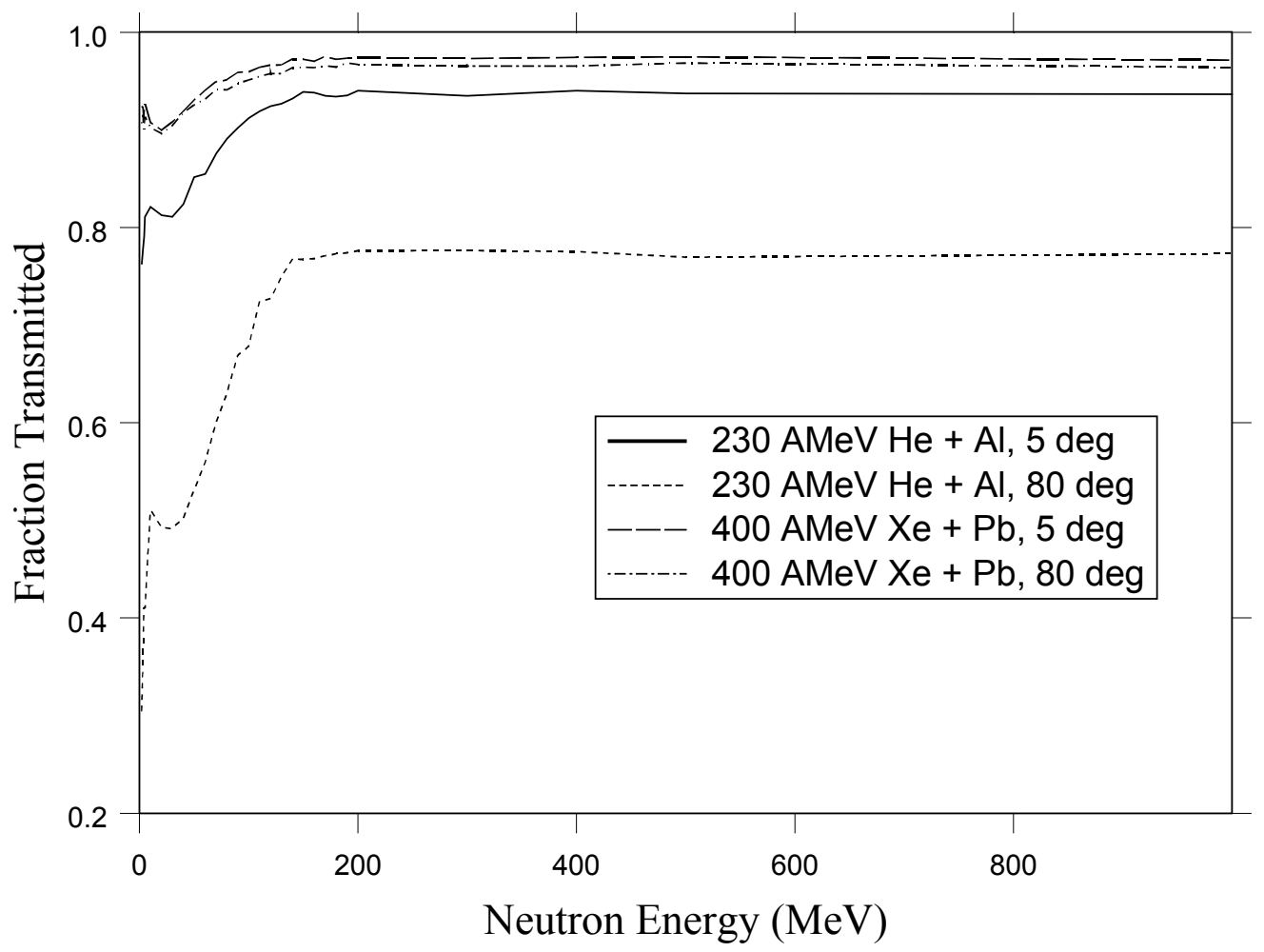

Fig. 2. The fraction of neutron flux transmitted from the target to the neutron detector as a function of neutron energy. Plots are shown for the four cases indicated in the legend. 


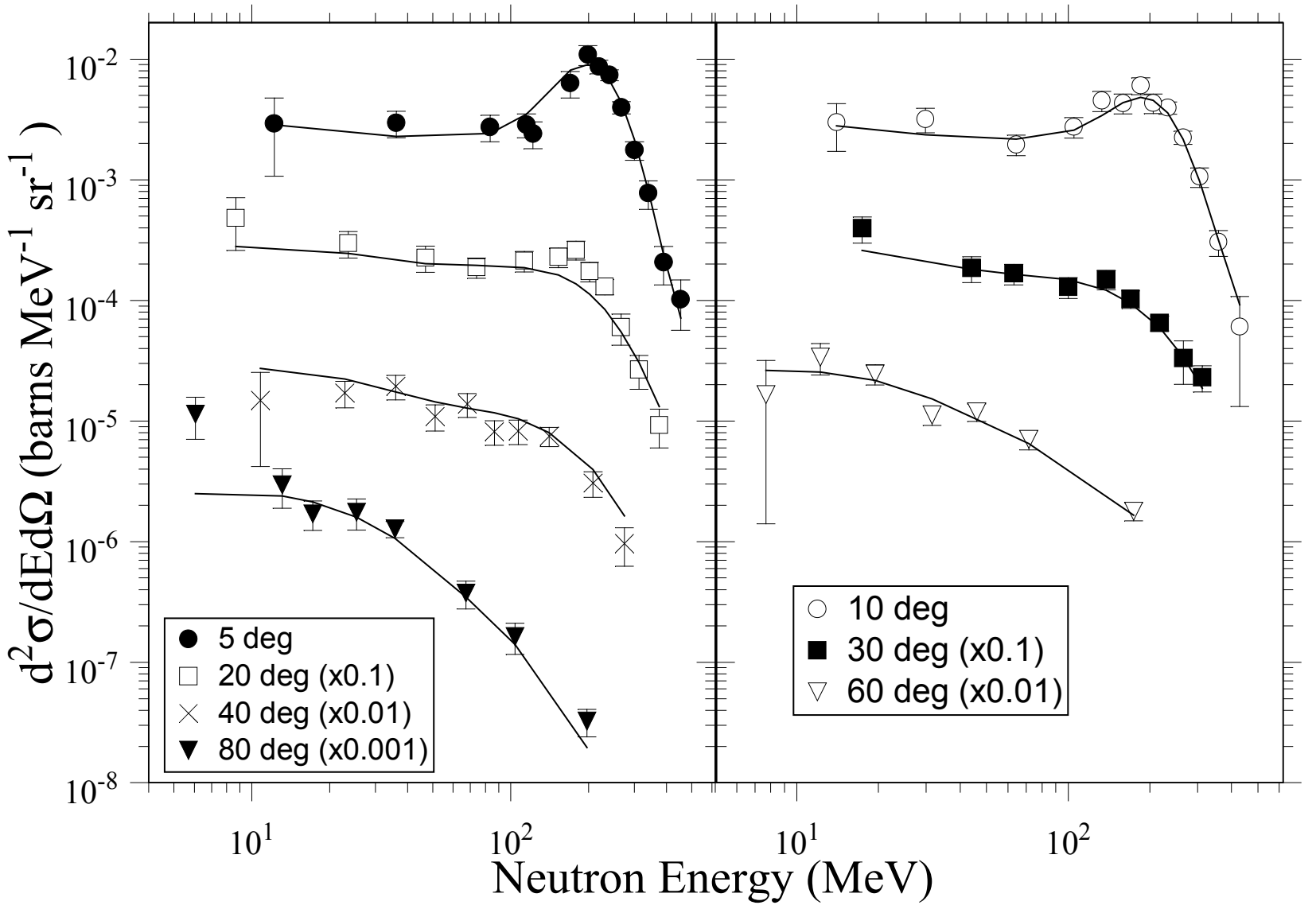

Fig. 3. Double-differential spectra from $230 \mathrm{MeV}$ /nucleon He interacting in an Al target. The spectra at each laboratory angle are offset by the indicated factors of 10 . The lines come from a moving-source fit described in the text. 


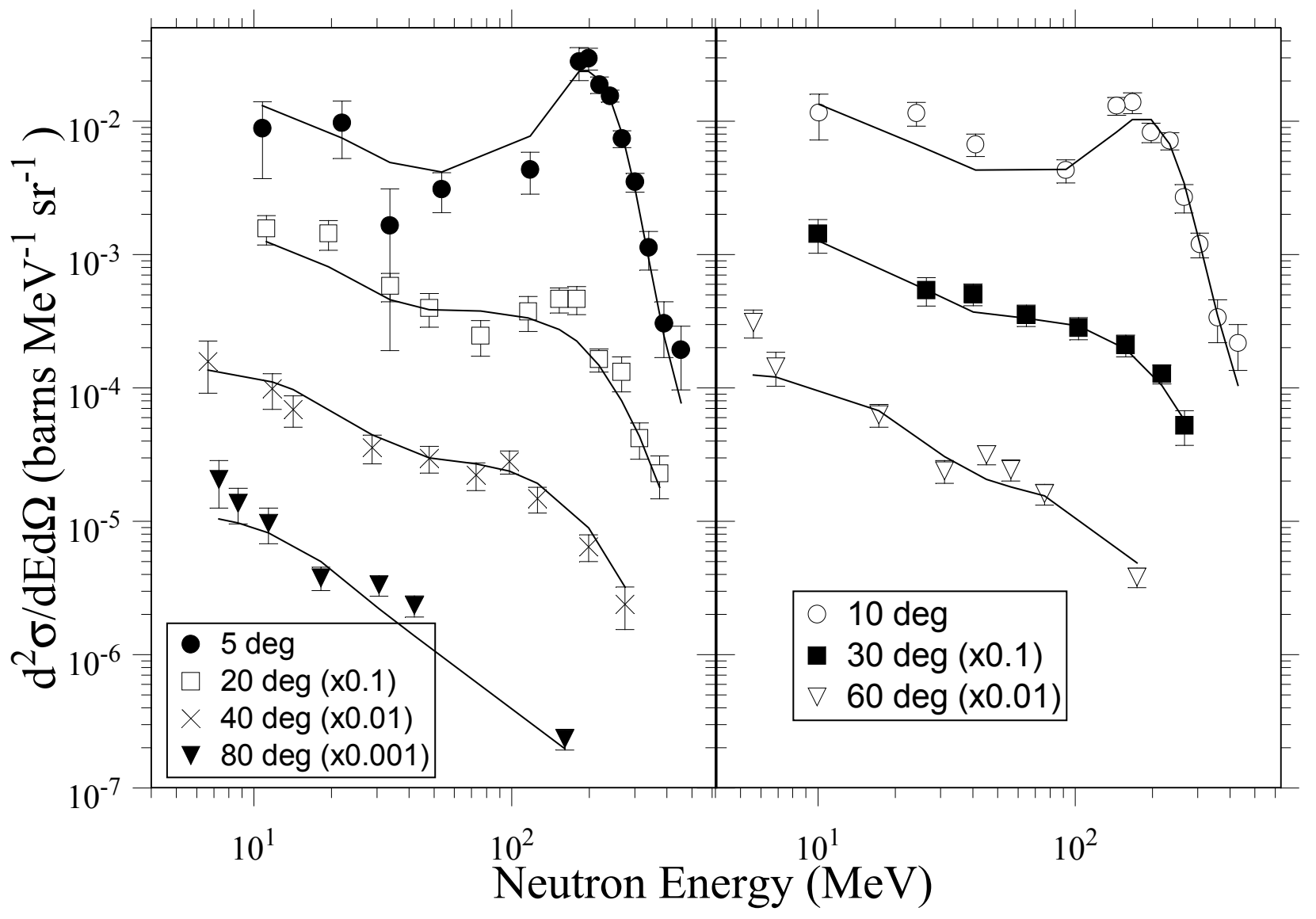

Fig. 4. Double-differential spectra from $230 \mathrm{MeV} /$ nucleon He interacting in a $\mathrm{Cu}$ target. The spectra at each laboratory angle are offset by the indicated factors of 10 . The lines come from a moving-source fit described in the text. 


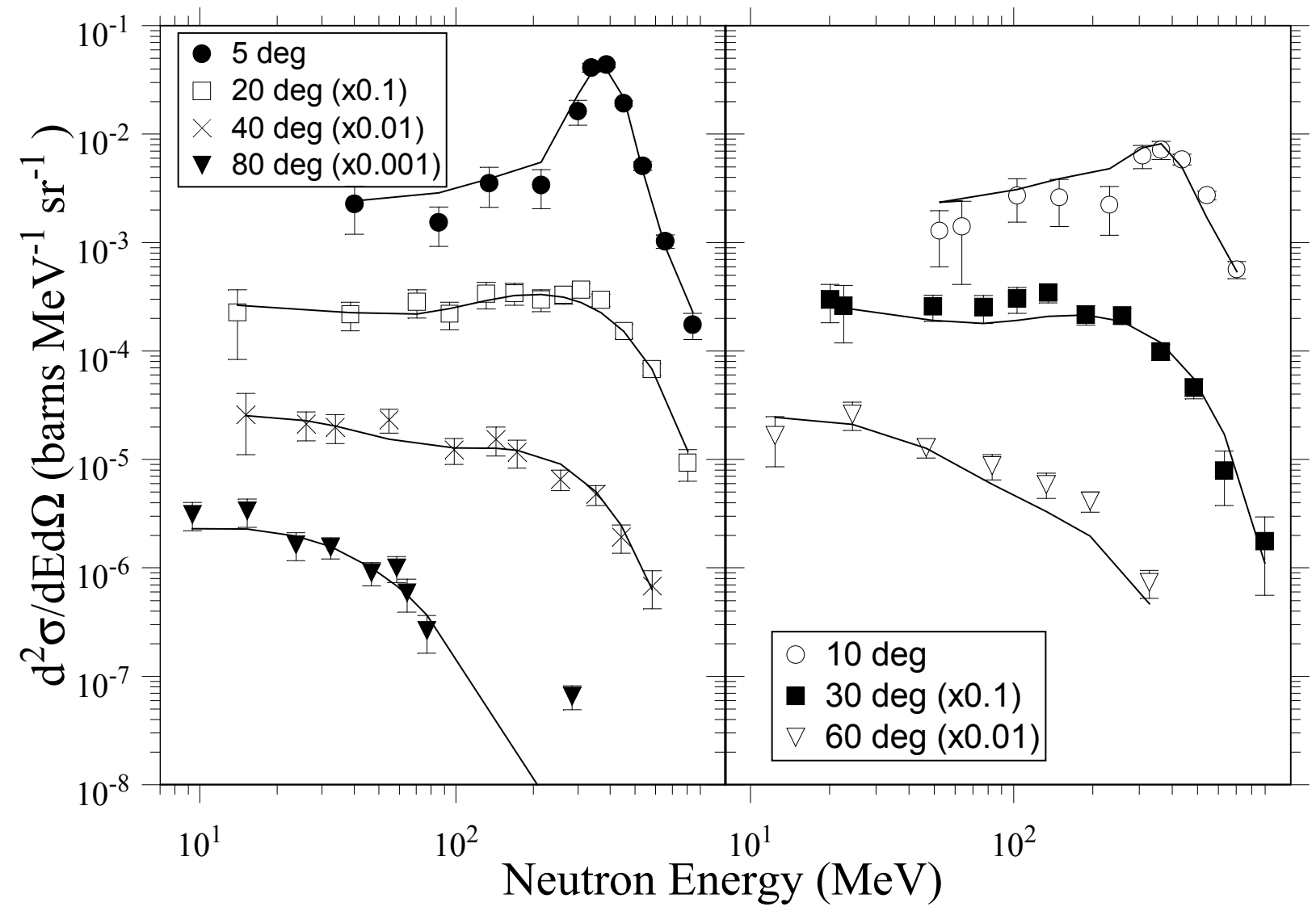

Fig. 5. Double-differential spectra from $400 \mathrm{MeV} /$ nucleon $\mathrm{N}$ interacting in a $\mathrm{C}$ target. The spectra at each laboratory angle are offset by the indicated factors of 10 . The lines come from a moving-source fit described in the text. 


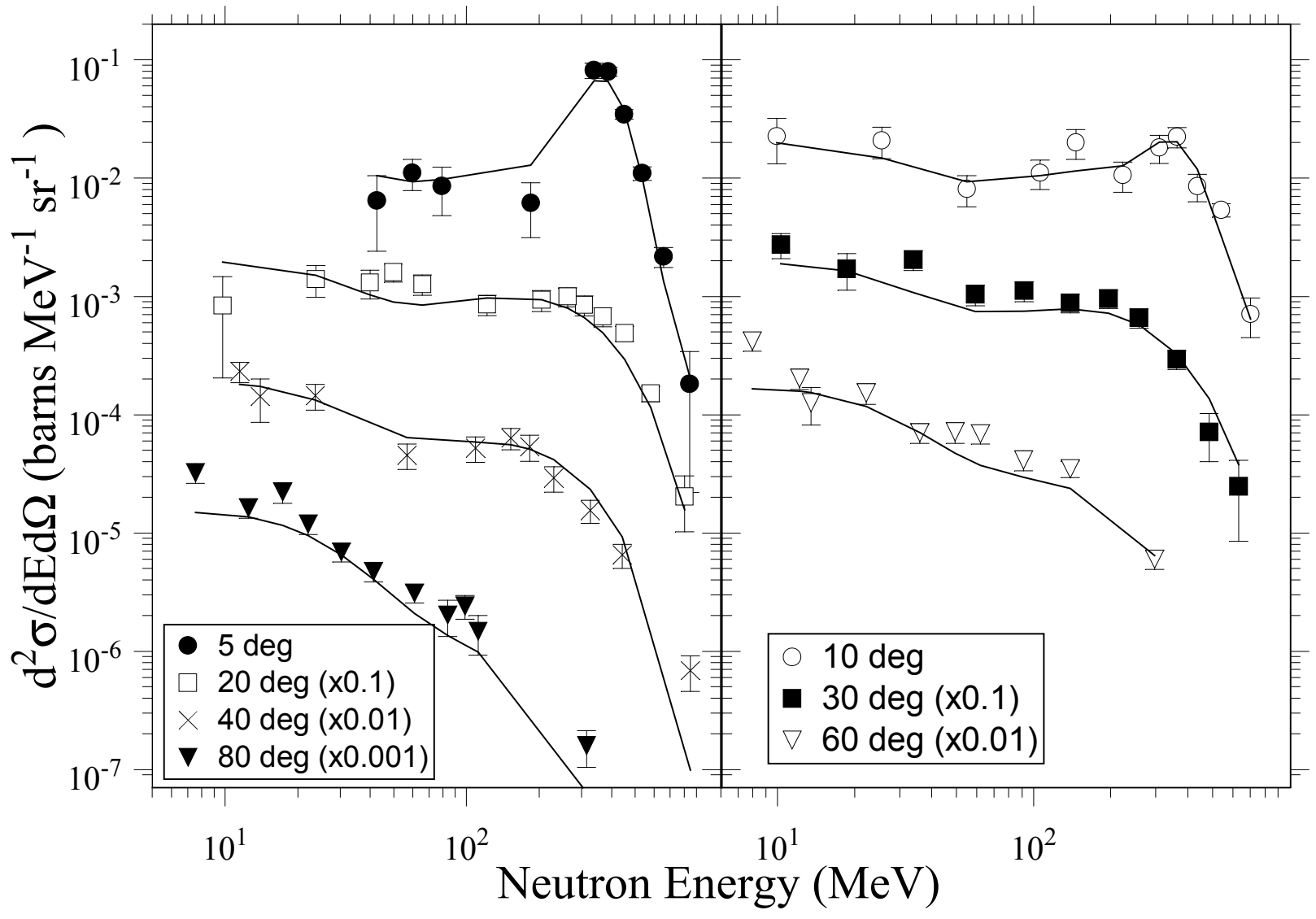

Fig. 6. Double-differential spectra from $400 \mathrm{MeV} /$ nucleon $\mathrm{N}$ interacting in a $\mathrm{Cu}$ target. The spectra at each laboratory angle are offset by the indicated factors of 10 . The lines come from a moving-source fit described in the text. 


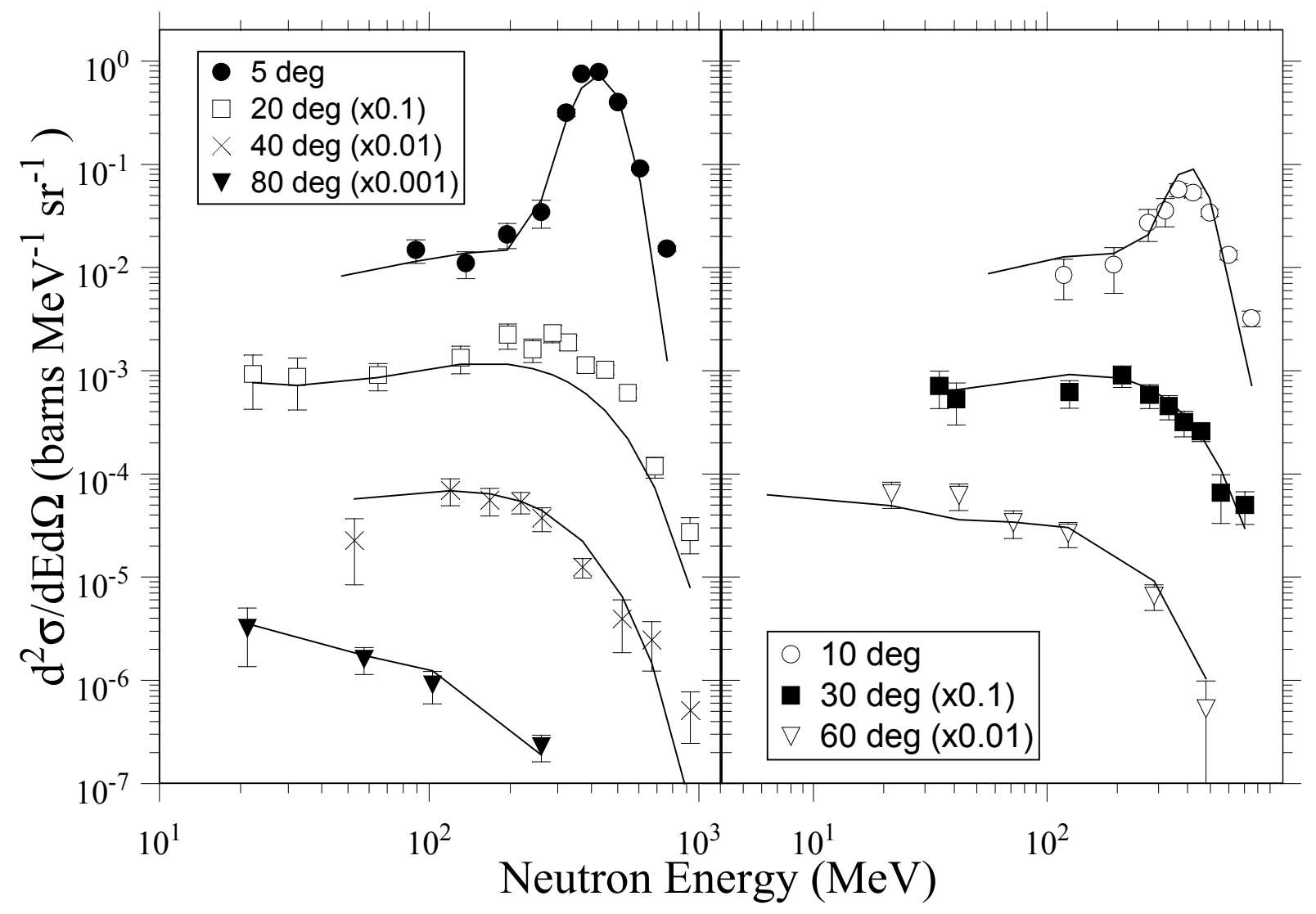

Fig. 7. Double-differential spectra from $400 \mathrm{MeV} /$ nucleon $\mathrm{Kr}$ interacting in a $\mathrm{Li}$ target. The spectra at each laboratory angle are offset by the indicated factors of 10 . The lines come from a moving-source fit described in the text. 


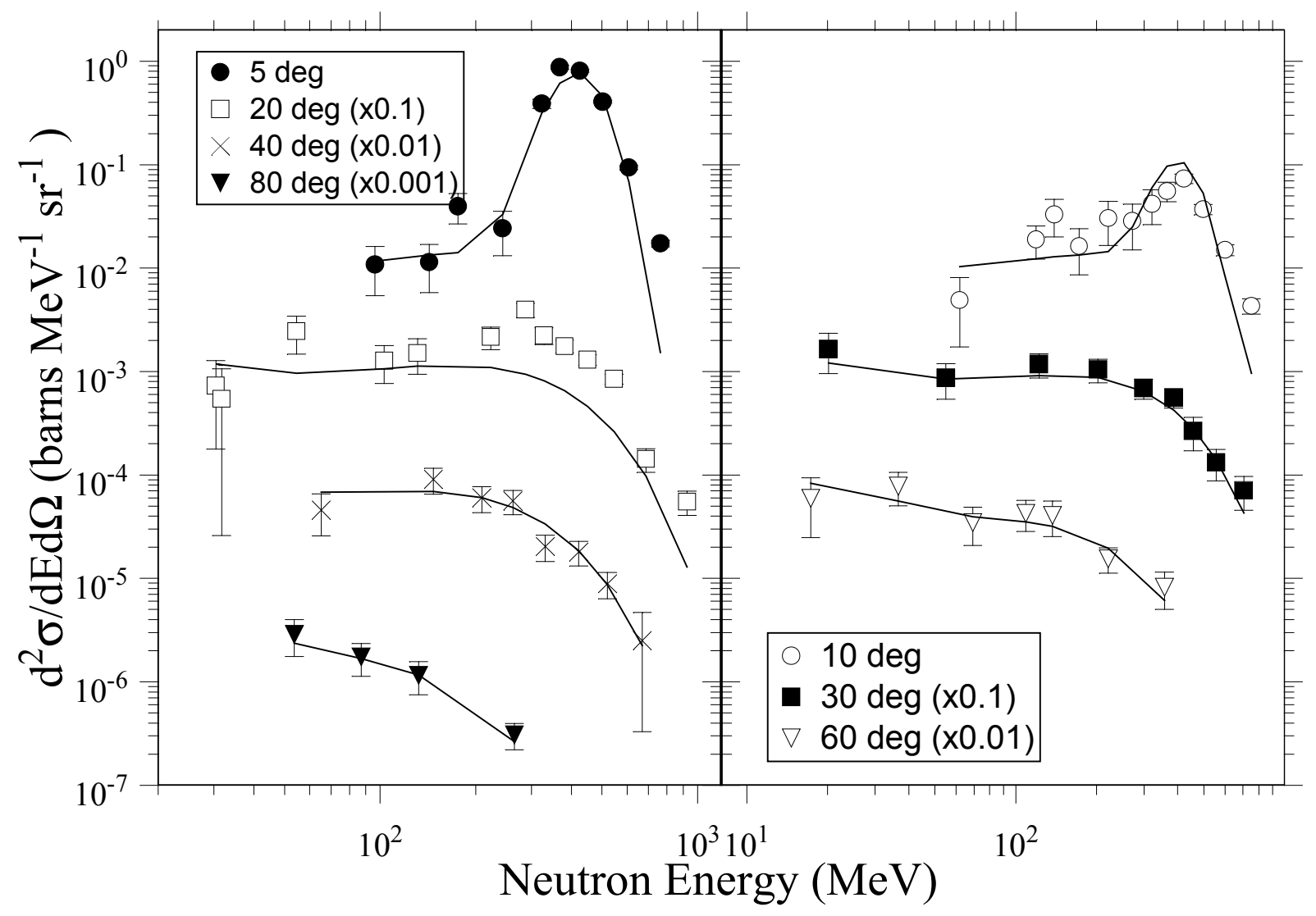

Fig. 8. Double-differential spectra from $400 \mathrm{MeV} /$ nucleon $\mathrm{Kr}$ interacting in a $\mathrm{C}$ target. The spectra at each laboratory angle are offset by the indicated factors of 10 . The lines come from a moving-source fit described in the text. 


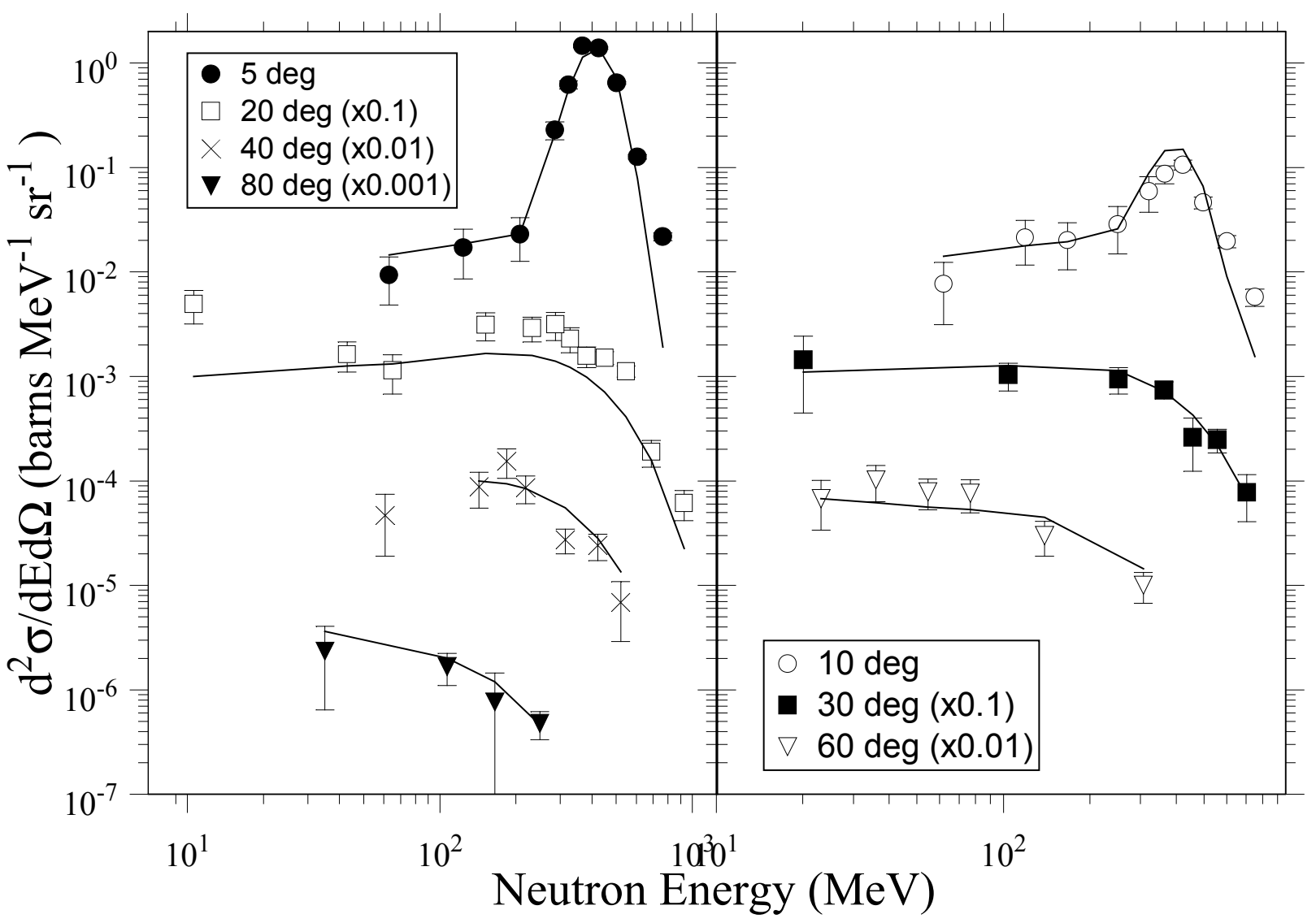

Fig. 9. Double-differential spectra from $400 \mathrm{MeV} /$ nucleon $\mathrm{Kr}$ interacting in a

$\mathrm{CH}_{2}$ target. The spectra at each laboratory angle are offset by the indicated factors of 10 .

The lines come from a moving-source fit described in the text. 


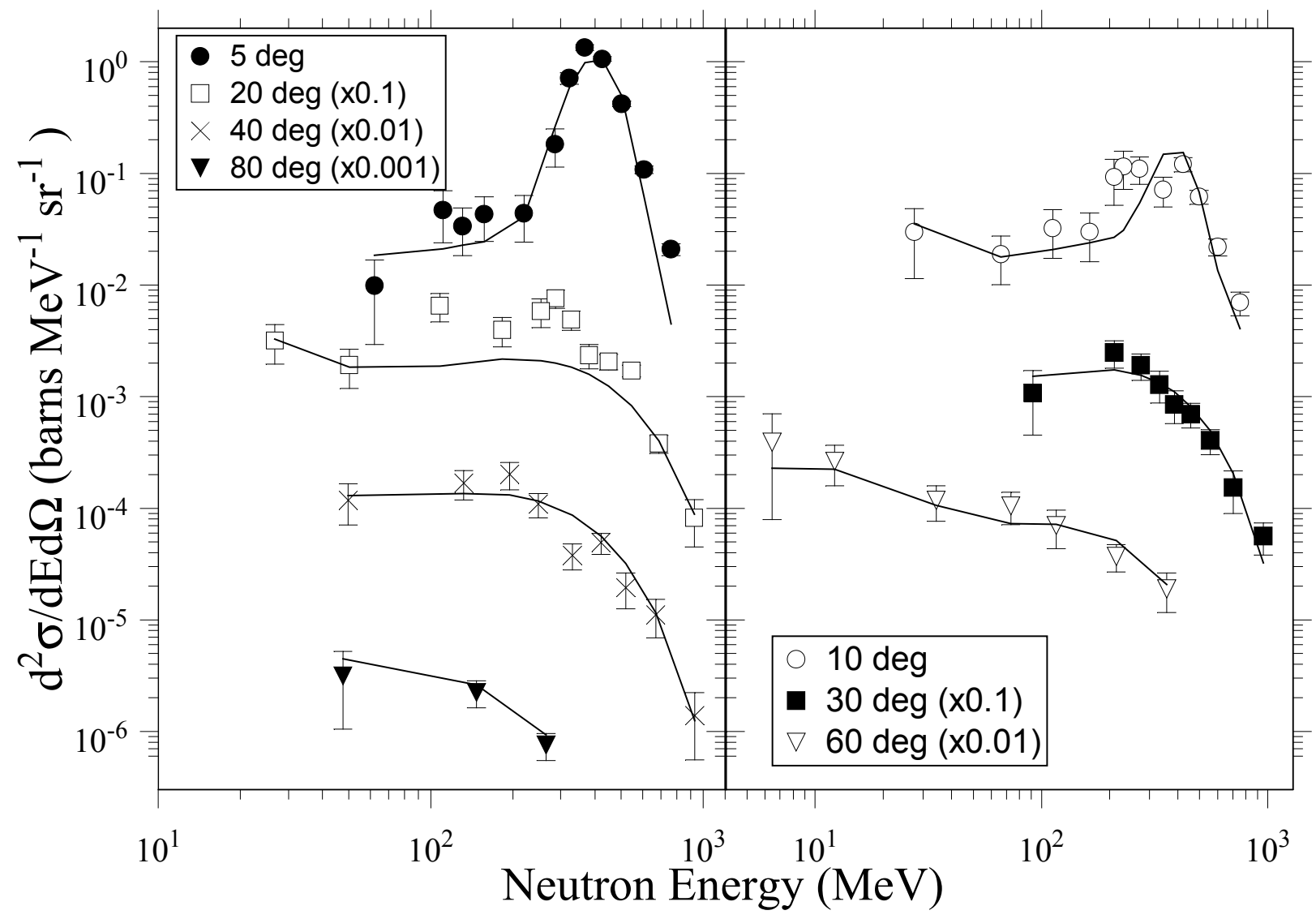

Fig. 10. Double-differential spectra from $400 \mathrm{MeV} /$ nucleon $\mathrm{Kr}$ interacting in an Al target. The spectra at each laboratory angle are offset by the indicated factors of 10 . The lines come from a moving-source fit described in the text. 


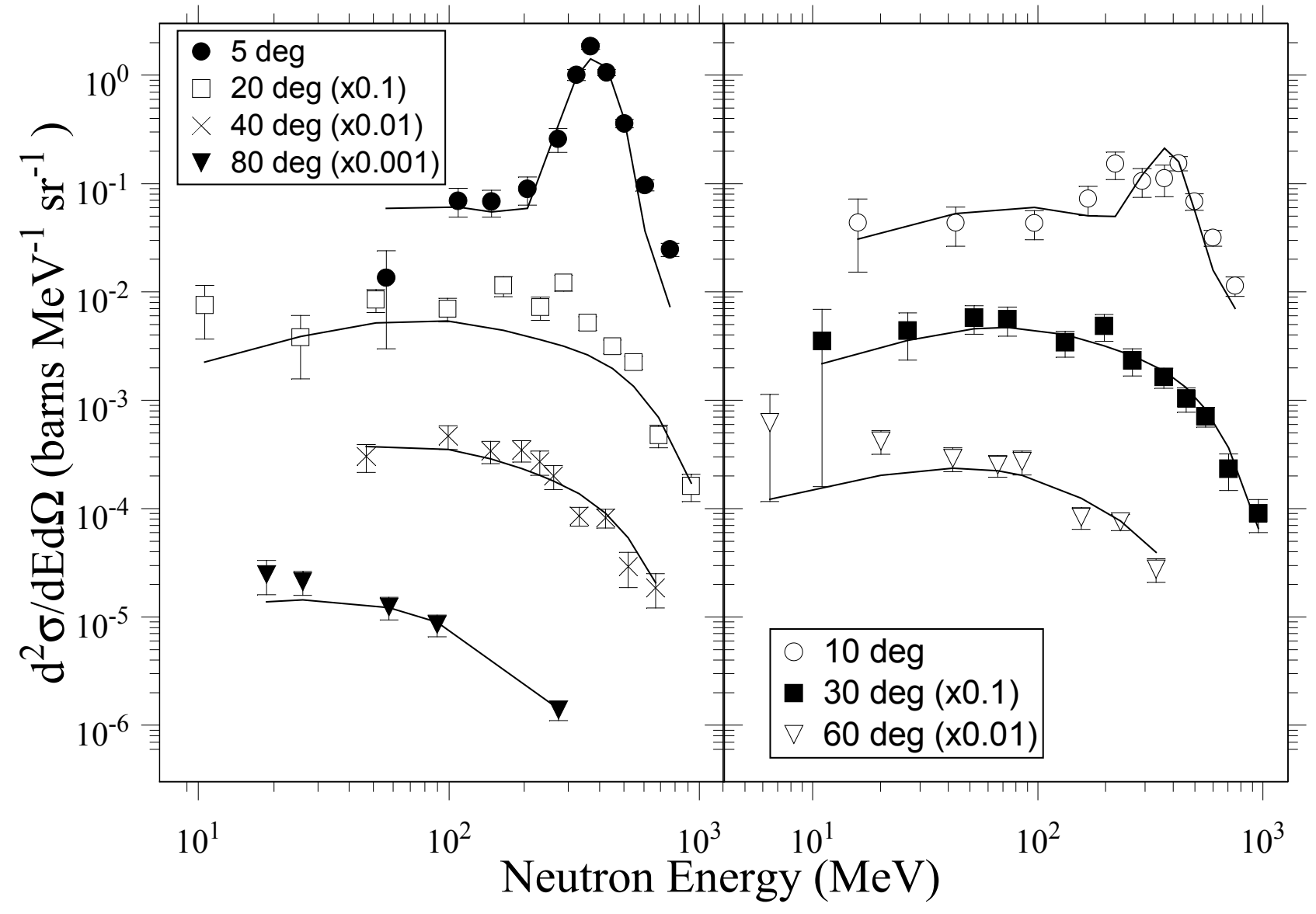

Fig. 11. Double-differential spectra from $400 \mathrm{MeV} /$ nucleon $\mathrm{Kr}$ interacting in a $\mathrm{Cu}$ target. The spectra at each laboratory angle are offset by the indicated factors of 10 . The lines come from a moving-source fit described in the text. 


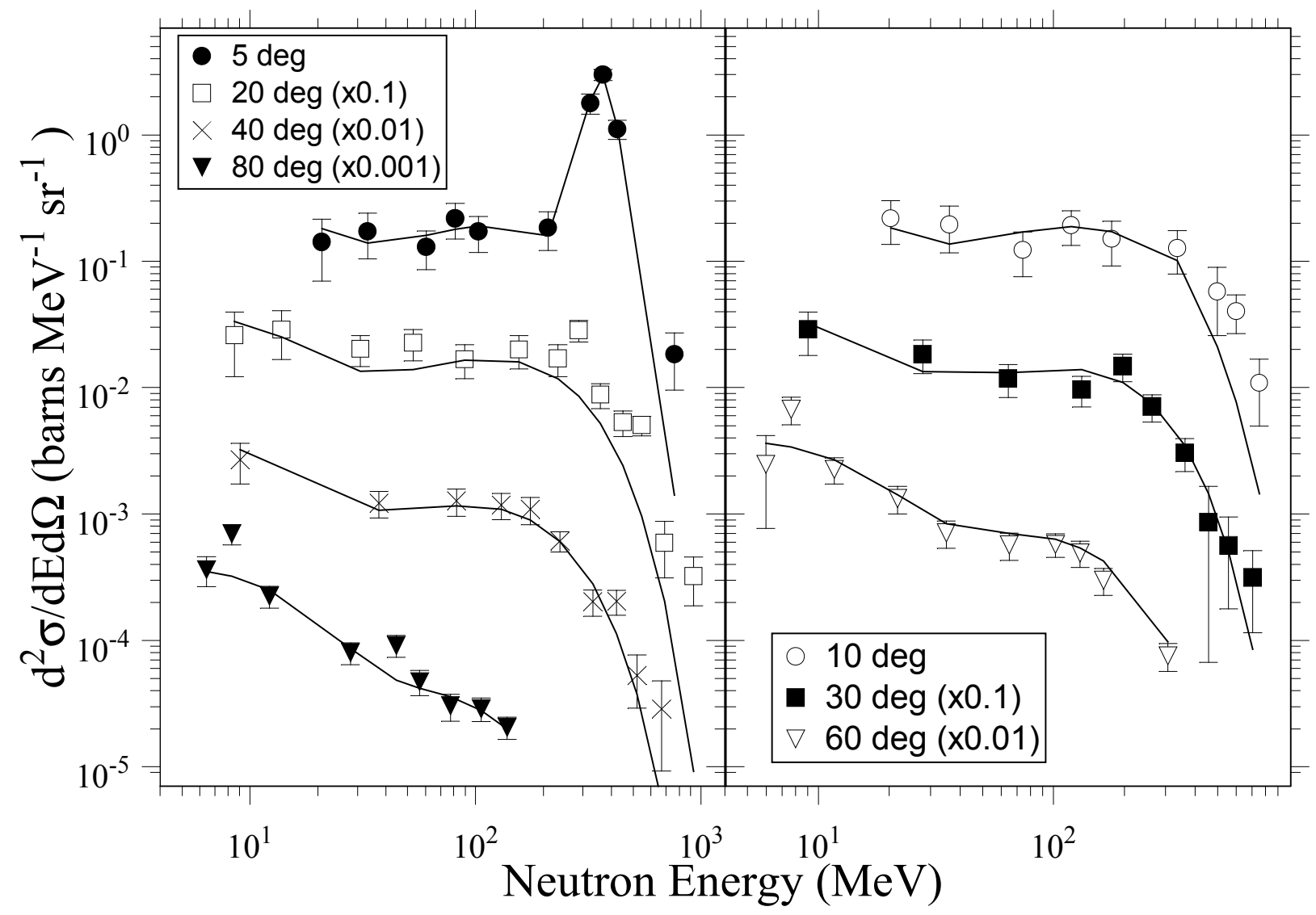

Fig. 12. Double-differential spectra from $400 \mathrm{MeV} /$ nucleon $\mathrm{Kr}$ interacting in a $\mathrm{Pb}$ target. The spectra at each laboratory angle are offset by the indicated factors of 10 . The lines come from a moving-source fit described in the text. 


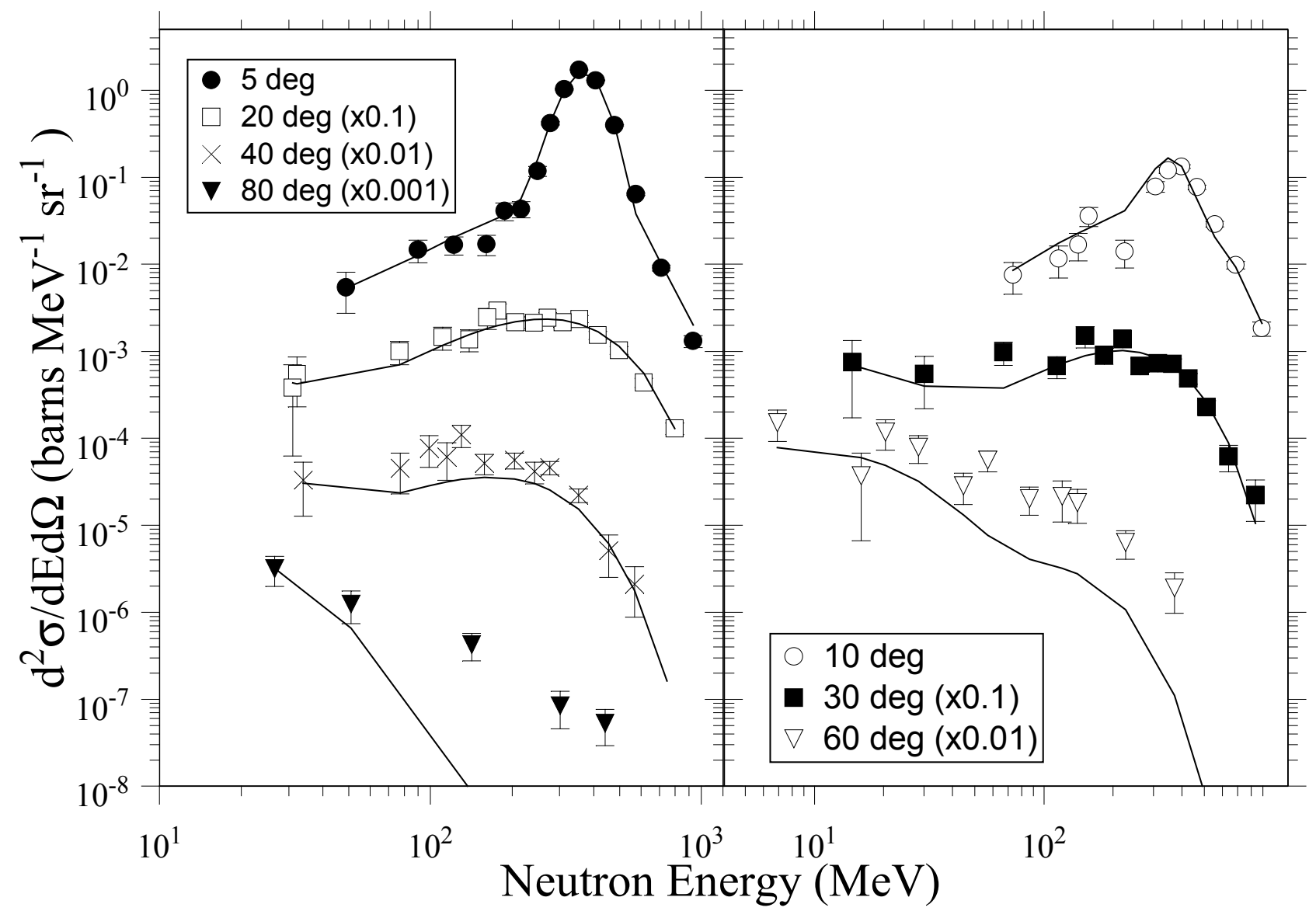

Fig. 13. Double-differential spectra from $400 \mathrm{MeV} /$ nucleon Xe interacting in a $\mathrm{Li}$ target. The spectra at each laboratory angle are offset by the indicated factors of 10 . The lines come from a moving-source fit described in the text. 


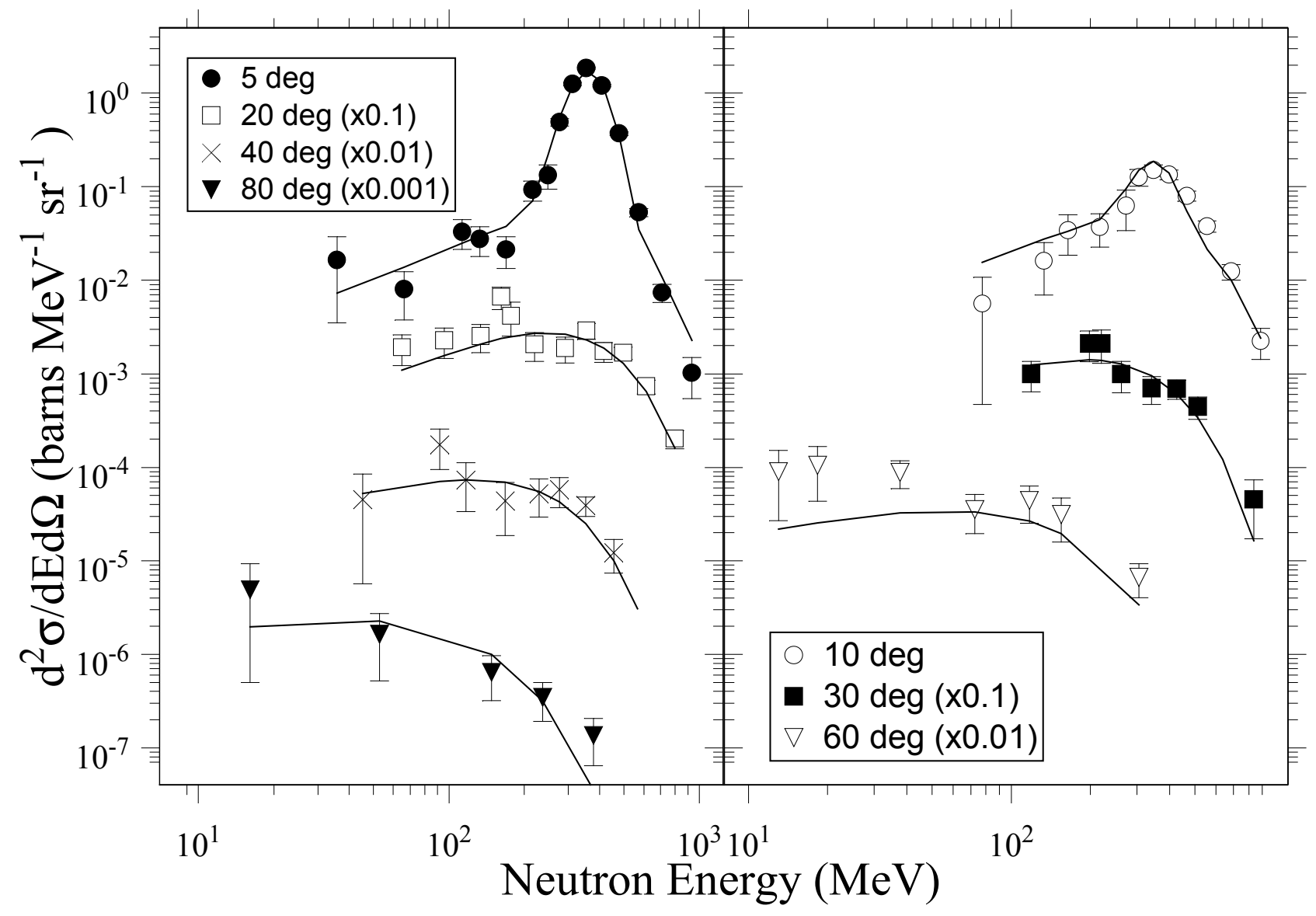

Fig. 14. Double-differential spectra from $400 \mathrm{MeV} /$ nucleon Xe interacting in a C target. The spectra at each laboratory angle are offset by the indicated factors of 10 . The lines come from a moving-source fit described in the text. 


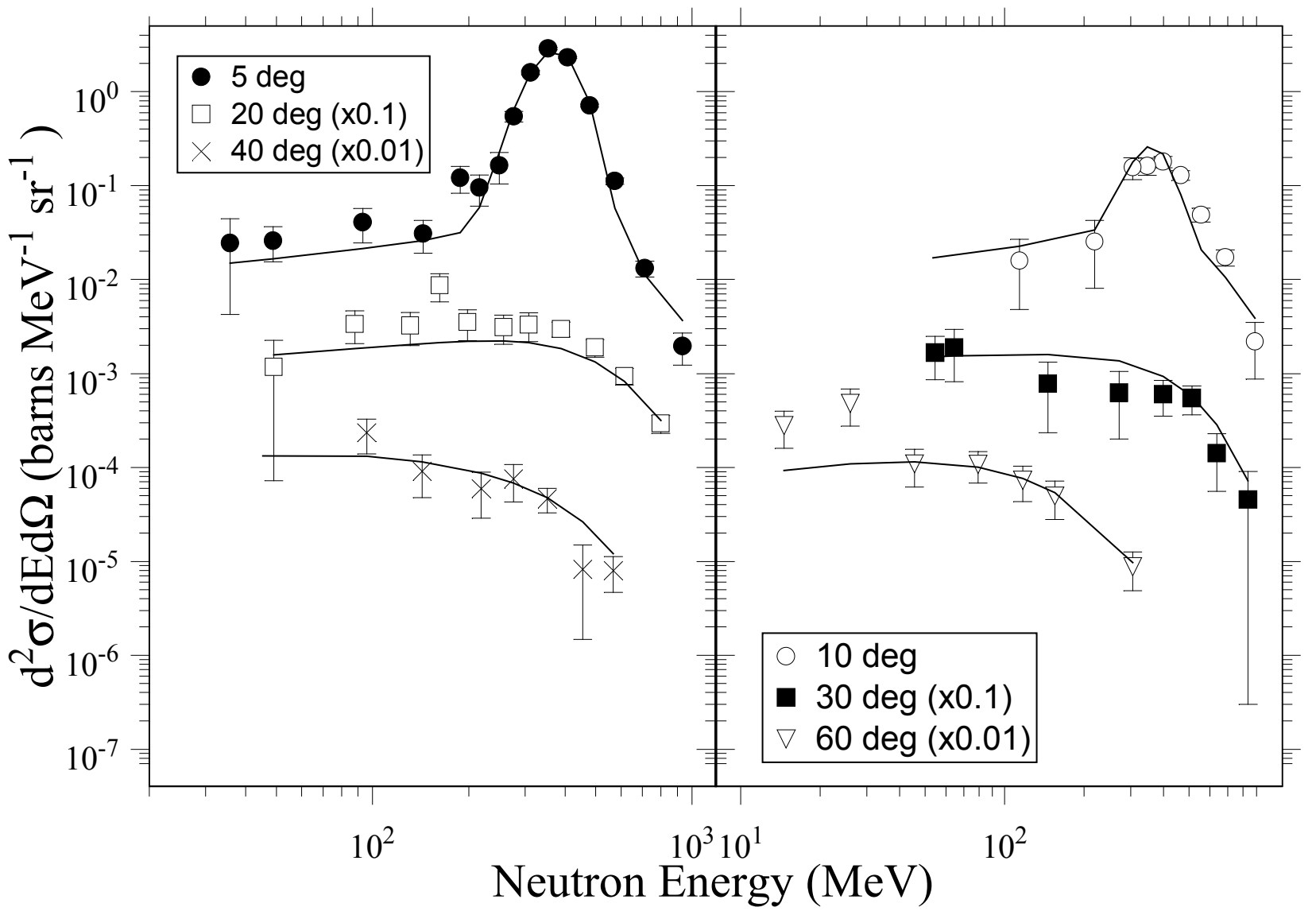

Fig. 15. Double-differential spectra from $400 \mathrm{MeV} /$ nucleon Xe interacting in a $\mathrm{CH}_{2}$ target. The spectra at each laboratory angle are offset by the indicated factors of 10 . The lines come from a moving-source fit described in the text. 


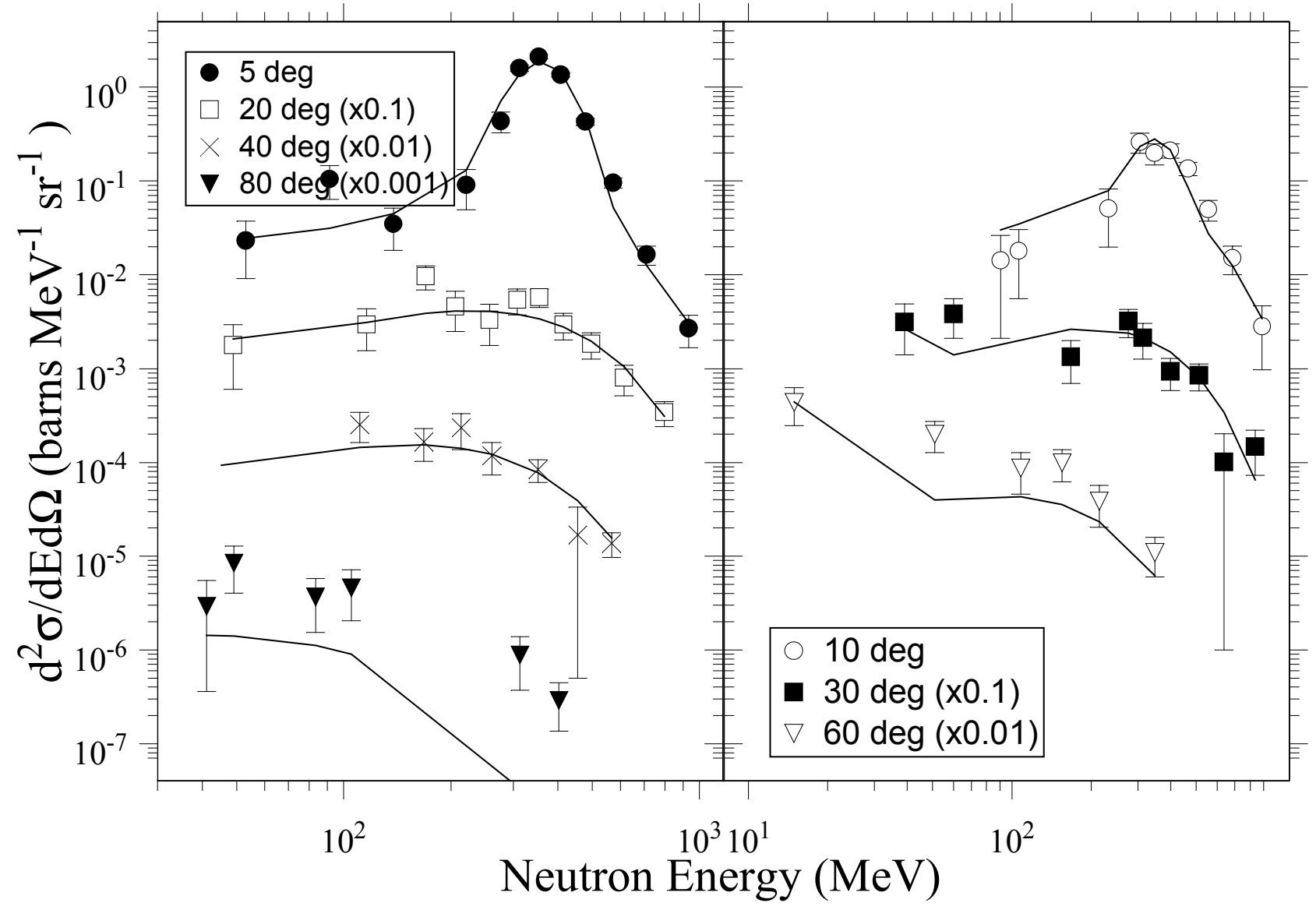

Fig. 16. Double-differential spectra from $400 \mathrm{MeV} /$ nucleon Xe interacting in an $\mathrm{Al}$ target. The spectra at each laboratory angle are offset by the indicated factors of 10 . The lines come from a moving-source fit described in the text. 


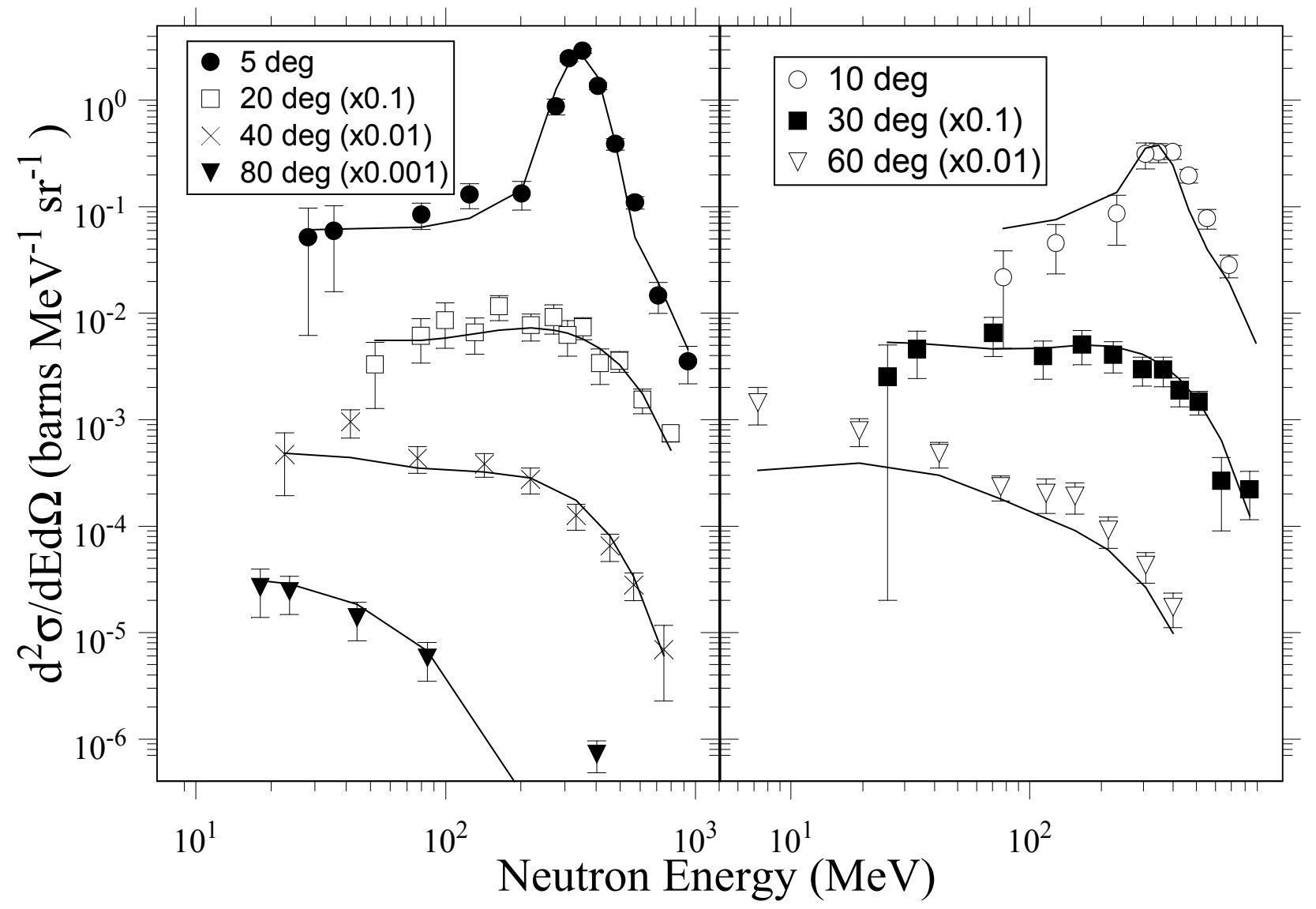

Fig. 17. Double-differential spectra from $400 \mathrm{MeV} /$ nucleon Xe interacting in a $\mathrm{Cu}$ target. The spectra at each laboratory angle are offset by the indicated factors of 10 . The lines come from a moving-source fit described in the text. 


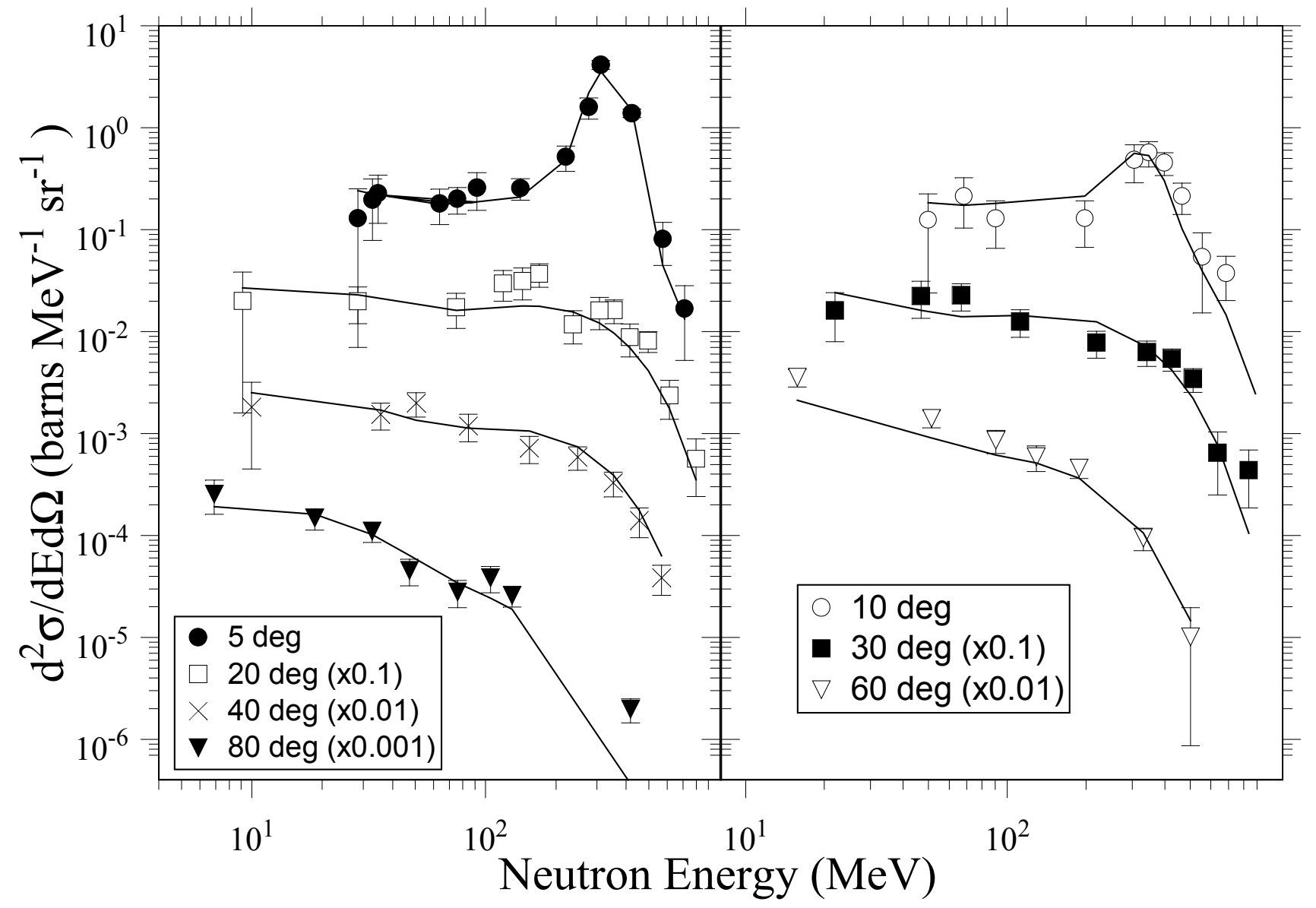

Fig. 18. Double-differential spectra from $400 \mathrm{MeV} /$ nucleon Xe interacting in a $\mathrm{Pb}$ target. The spectra at each laboratory angle are offset by the indicated factors of 10 . The lines come from a moving-source fit described in the text. 


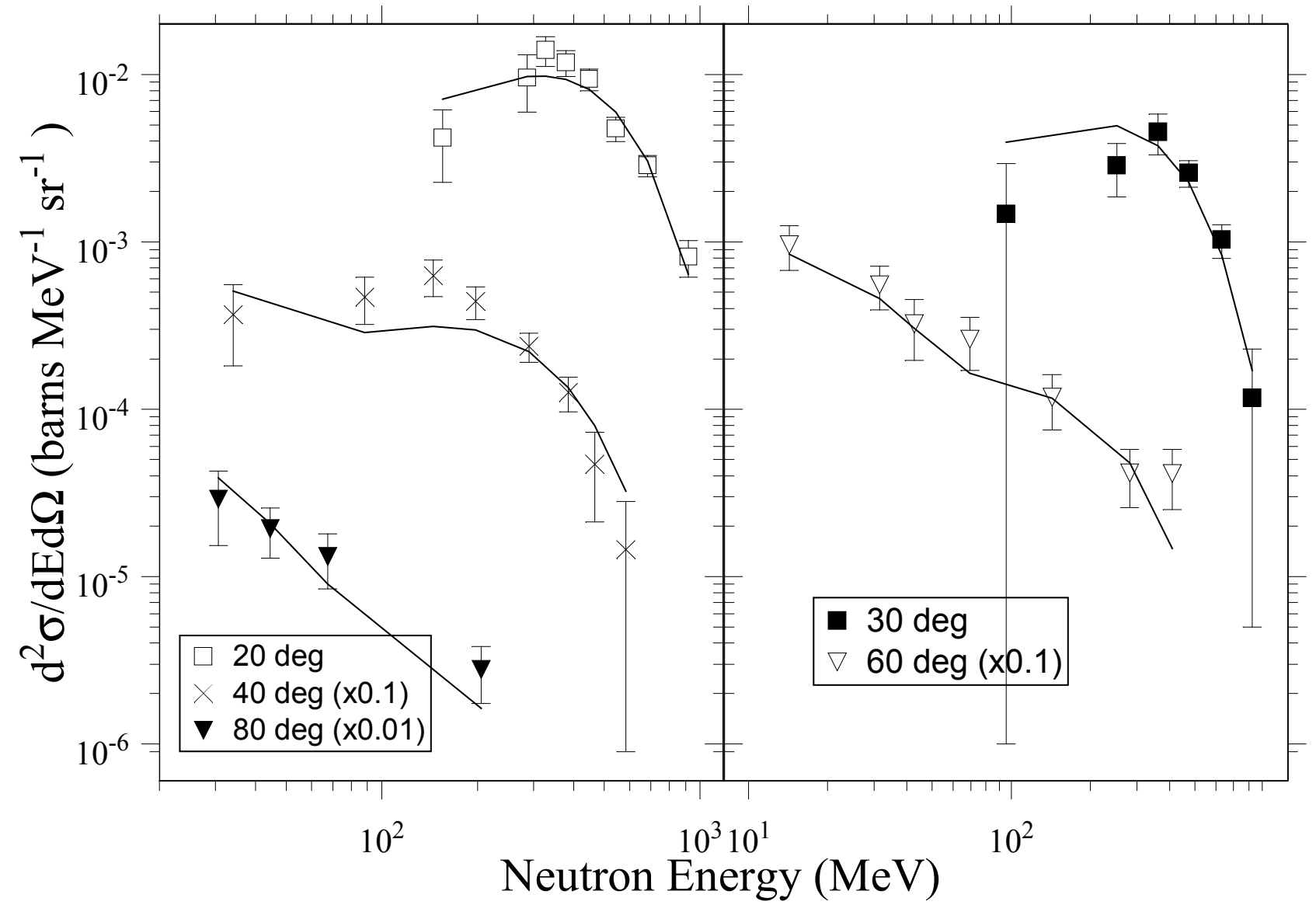

Fig. 19. Double-differential spectra from $500 \mathrm{MeV} /$ nucleon Fe interacting in a Li target. The spectra at each laboratory angle are offset by the indicated factors of 10 . The lines come from a moving-source fit described in the text. 


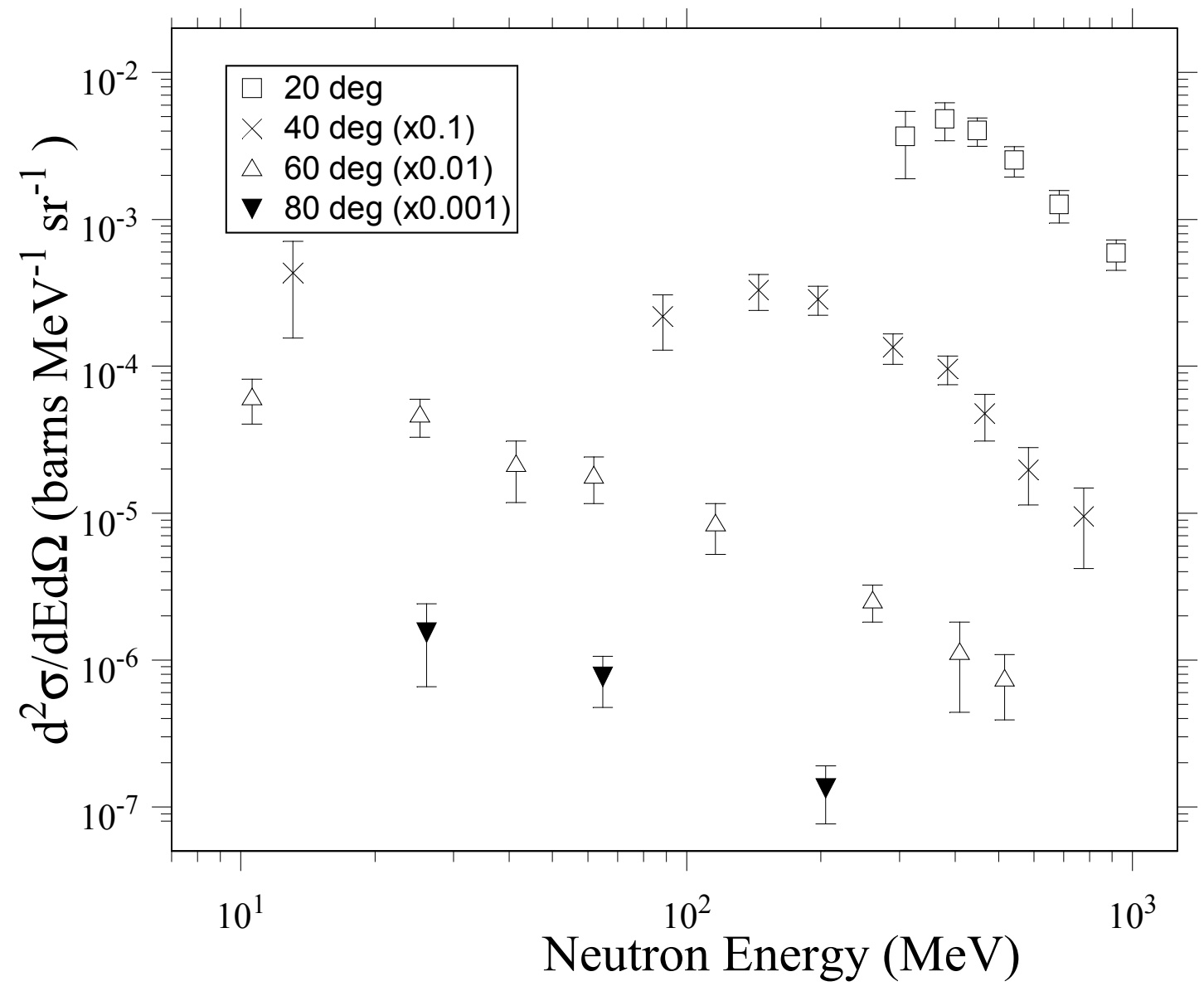

Fig. 20. Double-differential spectra from $500 \mathrm{MeV} /$ nucleon Fe interacting in a $\mathrm{CH}_{2}$ target. The spectra at each laboratory angle are offset by the indicated factors of 10 . 


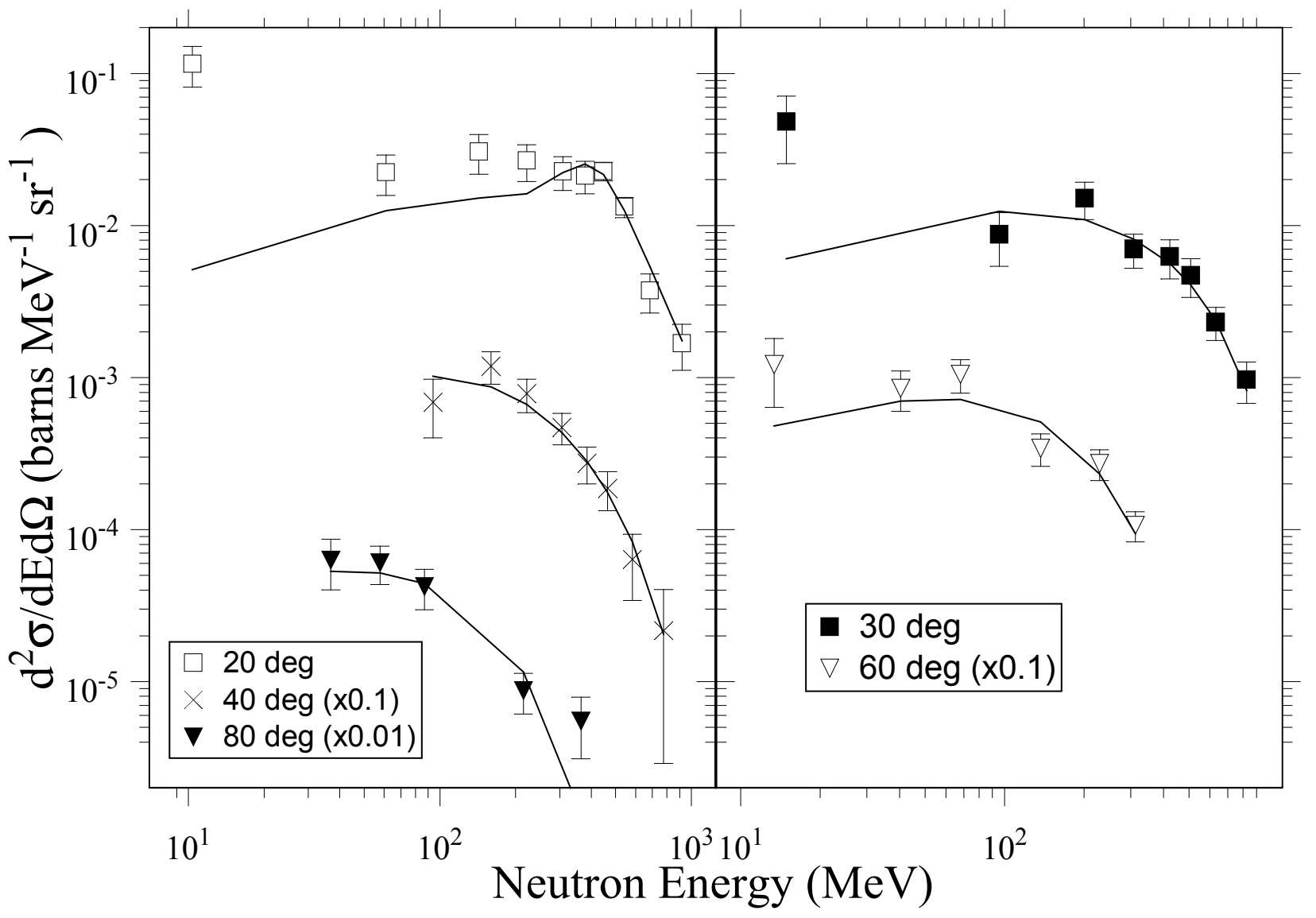

Fig. 21. Double-differential spectra from $500 \mathrm{MeV} /$ nucleon Fe interacting in an $\mathrm{Al}$ target. The spectra at each laboratory angle are offset by the indicated factors of 10 . The lines come from a moving-source fit described in the text. 


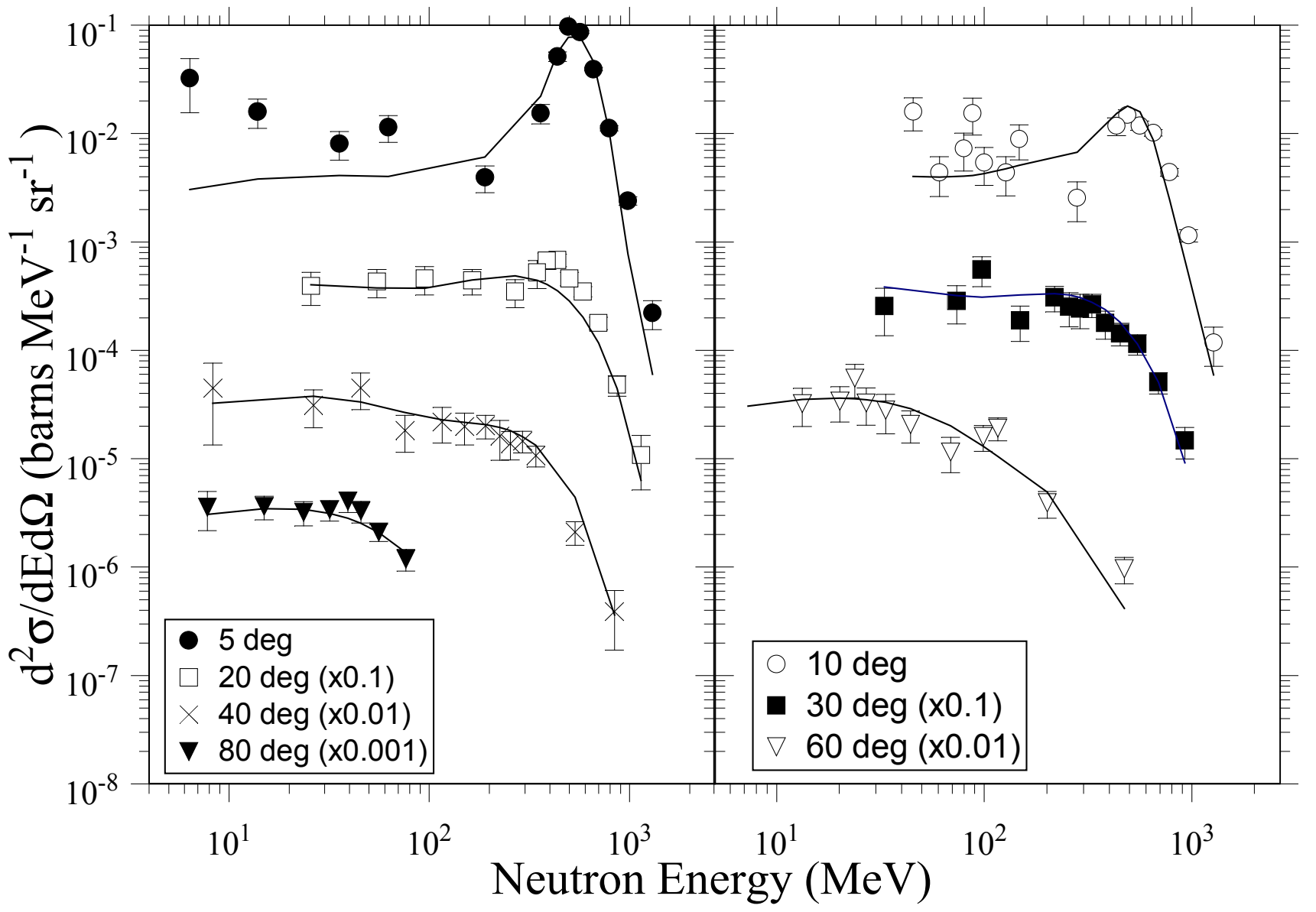

Fig. 22. Double-differential spectra from $600 \mathrm{MeV}$ /nucleon Si interacting in a C target. The spectra at each laboratory angle are offset by the indicated factors of 10 . The lines come from a moving-source fit described in the text. 


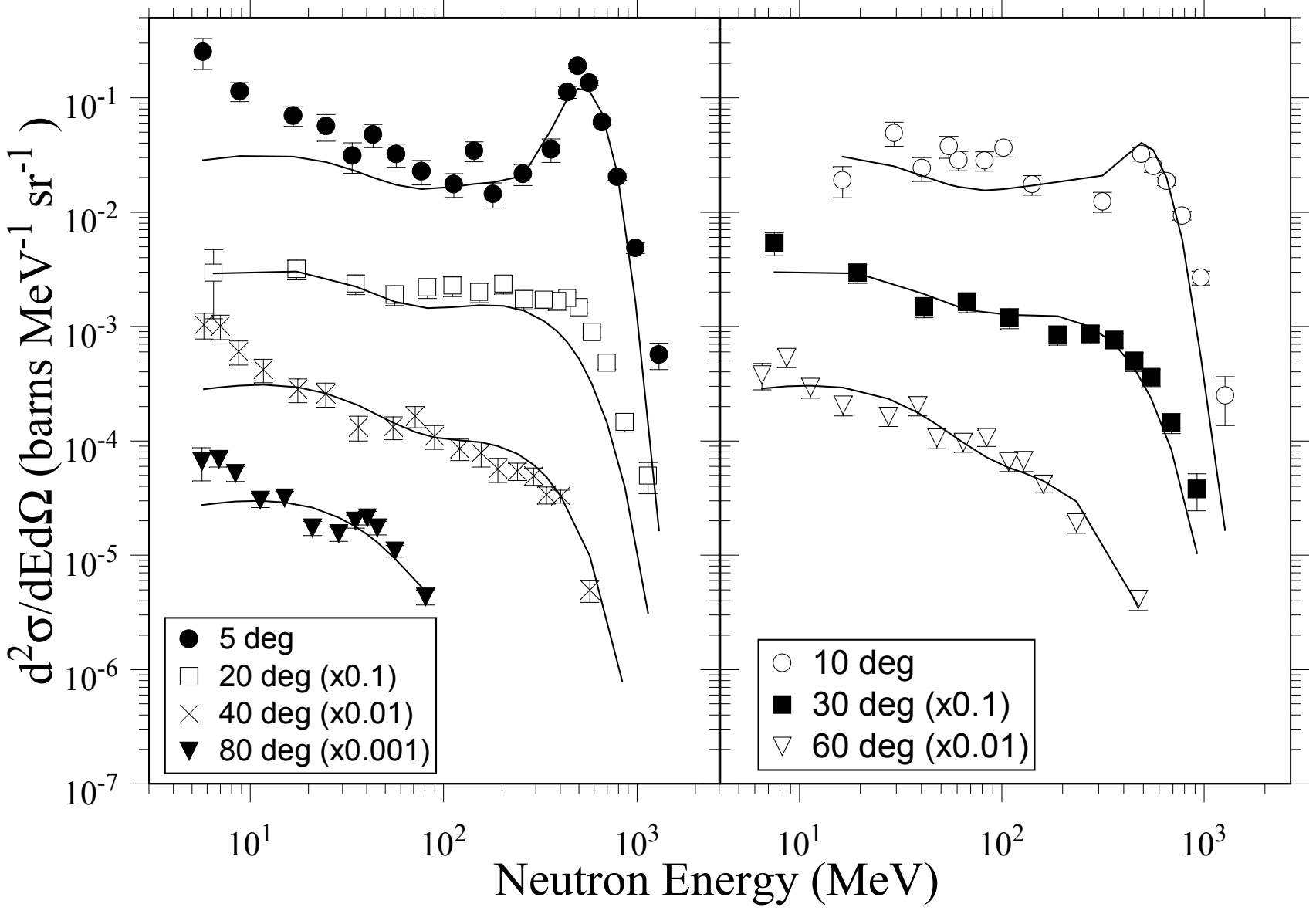

Fig. 23. Double-differential spectra from $600 \mathrm{MeV} /$ nucleon $\mathrm{Si}$ interacting in a $\mathrm{Cu}$ target. The spectra at each laboratory angle are offset by the indicated factors of 10 . The lines come from a moving-source fit described in the text. 


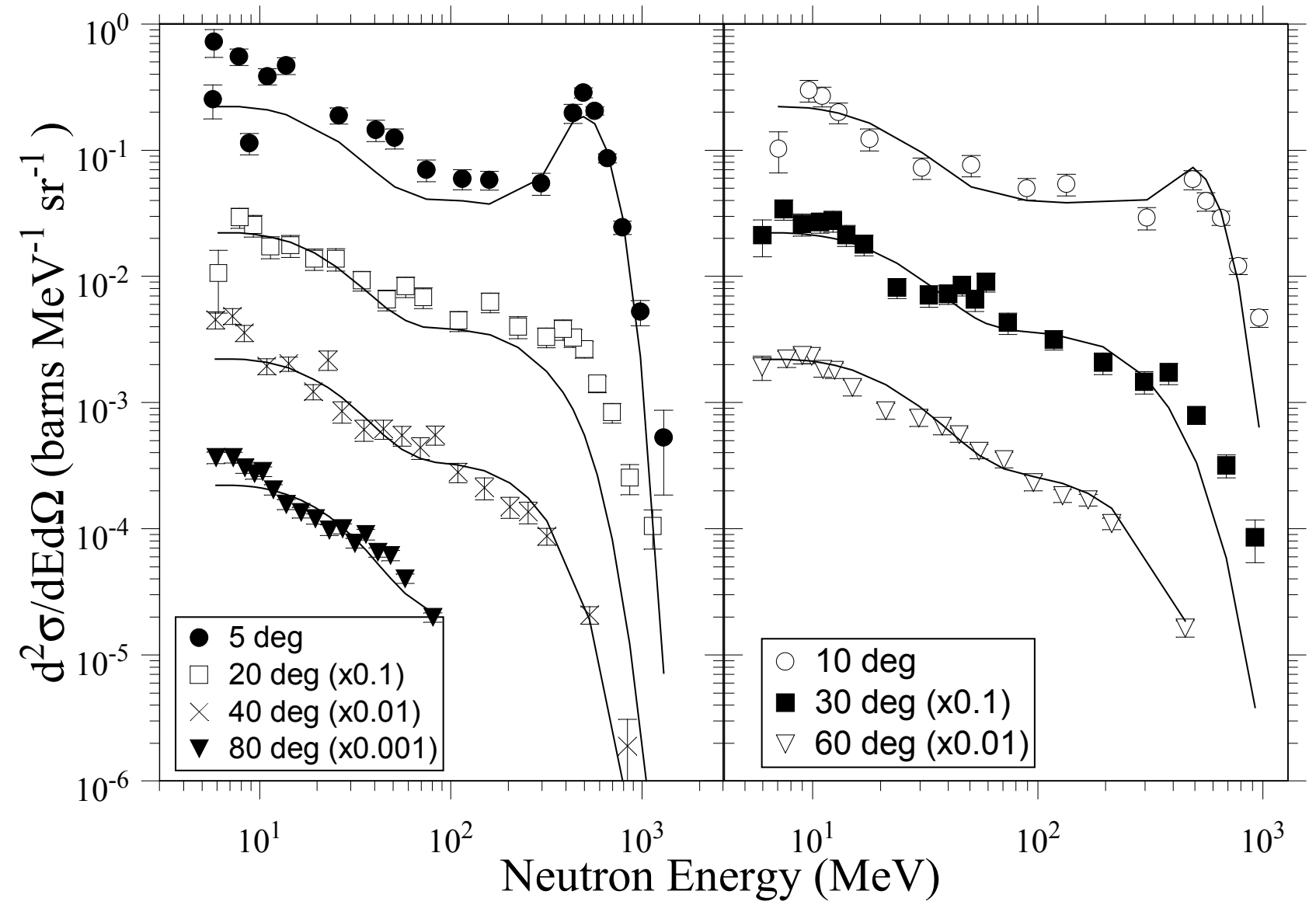

Fig. 24. Double-differential spectra from $600 \mathrm{MeV} /$ nucleon Si interacting in a $\mathrm{Pb}$ target. The spectra at each laboratory angle are offset by the indicated factors of 10 . The lines come from a moving-source fit described in the text. 


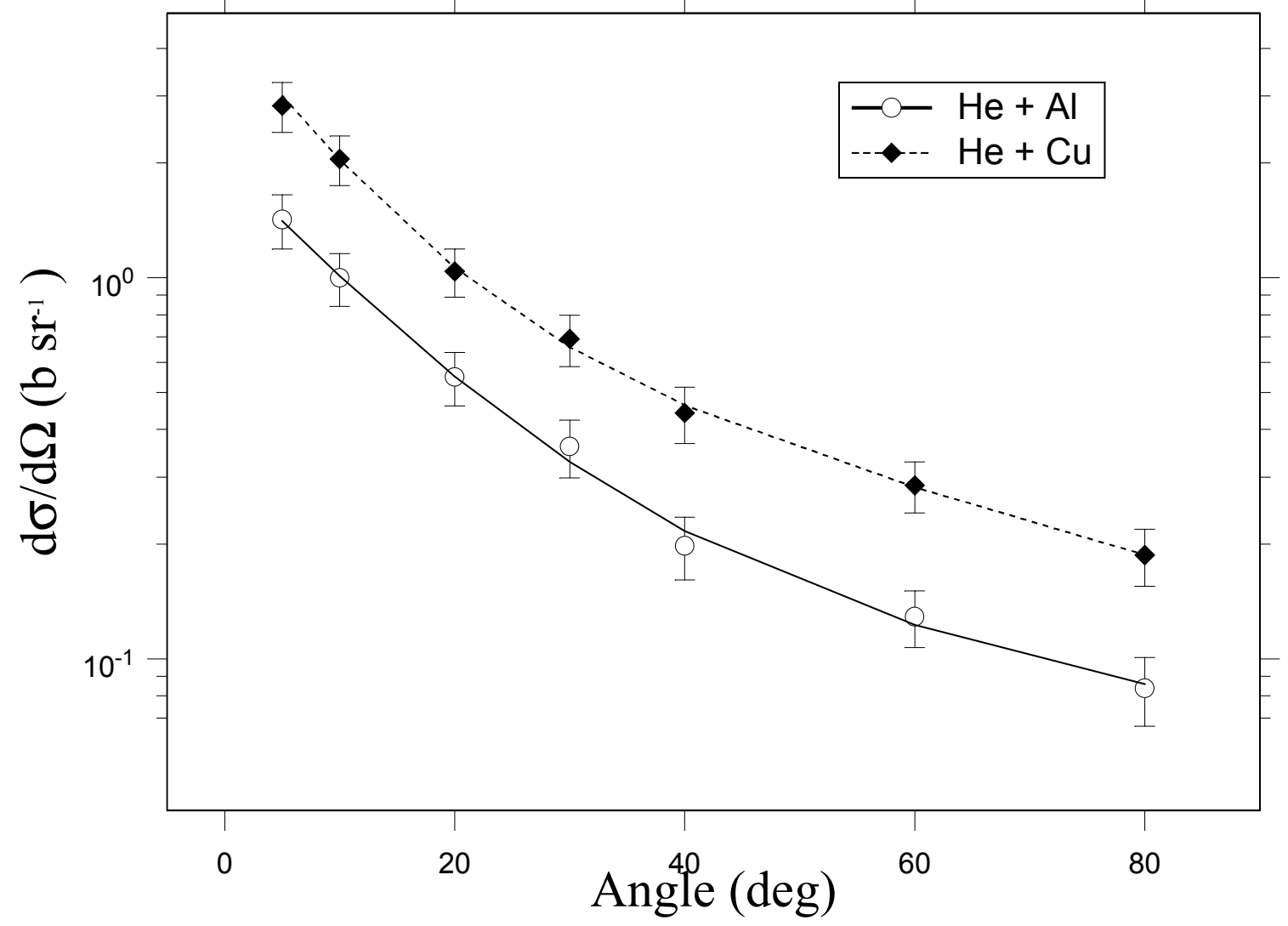

Fig. 25. Angular distribution from the $230 \mathrm{MeV} /$ nucleon $\mathrm{He}$ systems for $\mathrm{E}_{\mathrm{n}}>10$ MeV. The lines show a fit to the data using Eqn. 5. 


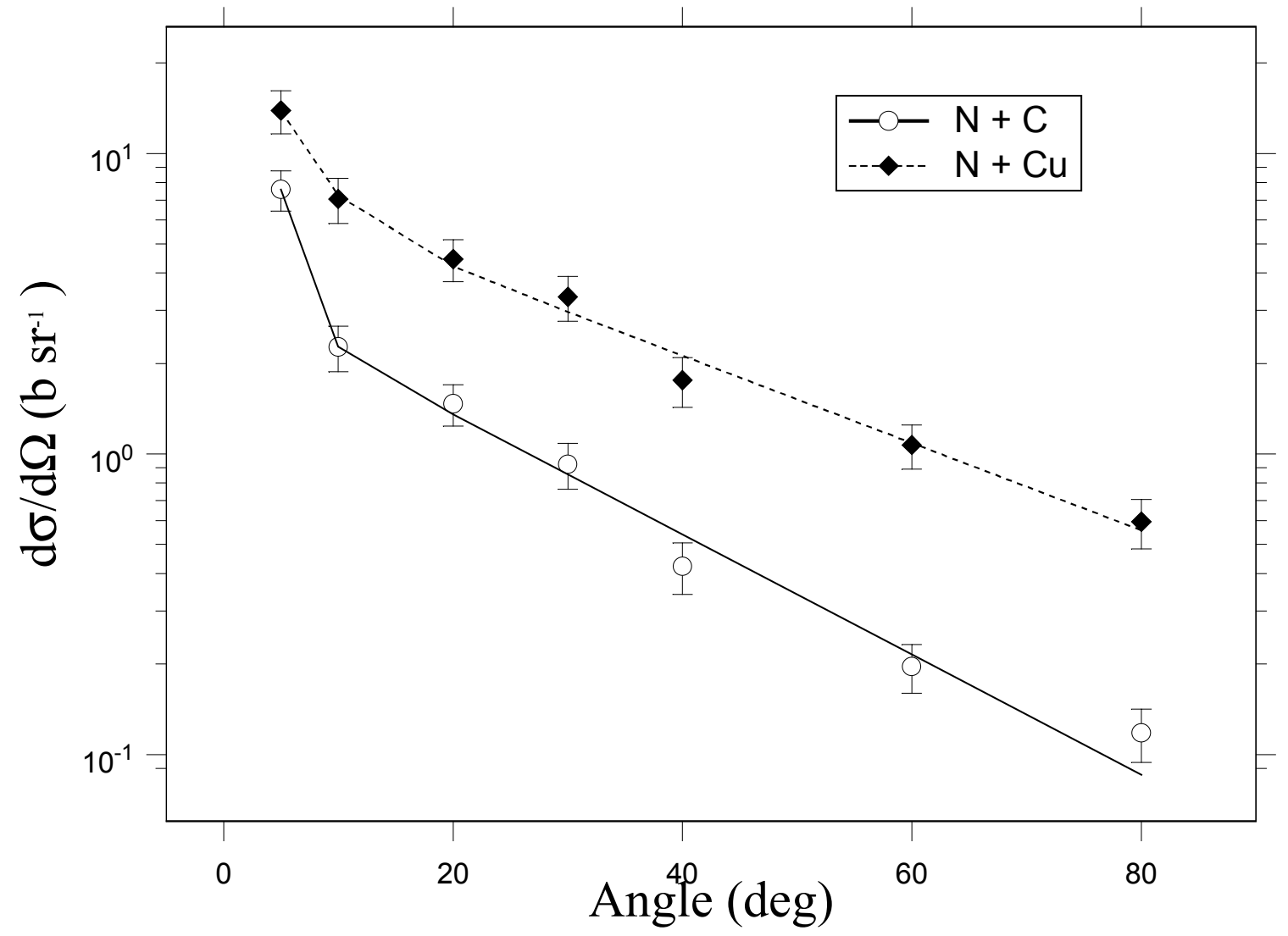

Fig. 26. Angular distribution from the $400 \mathrm{MeV} /$ nucleon $\mathrm{N}$ systems for $\mathrm{E}_{\mathrm{n}}>10$ MeV. The lines show a fit to the data using Eqn. 5. 


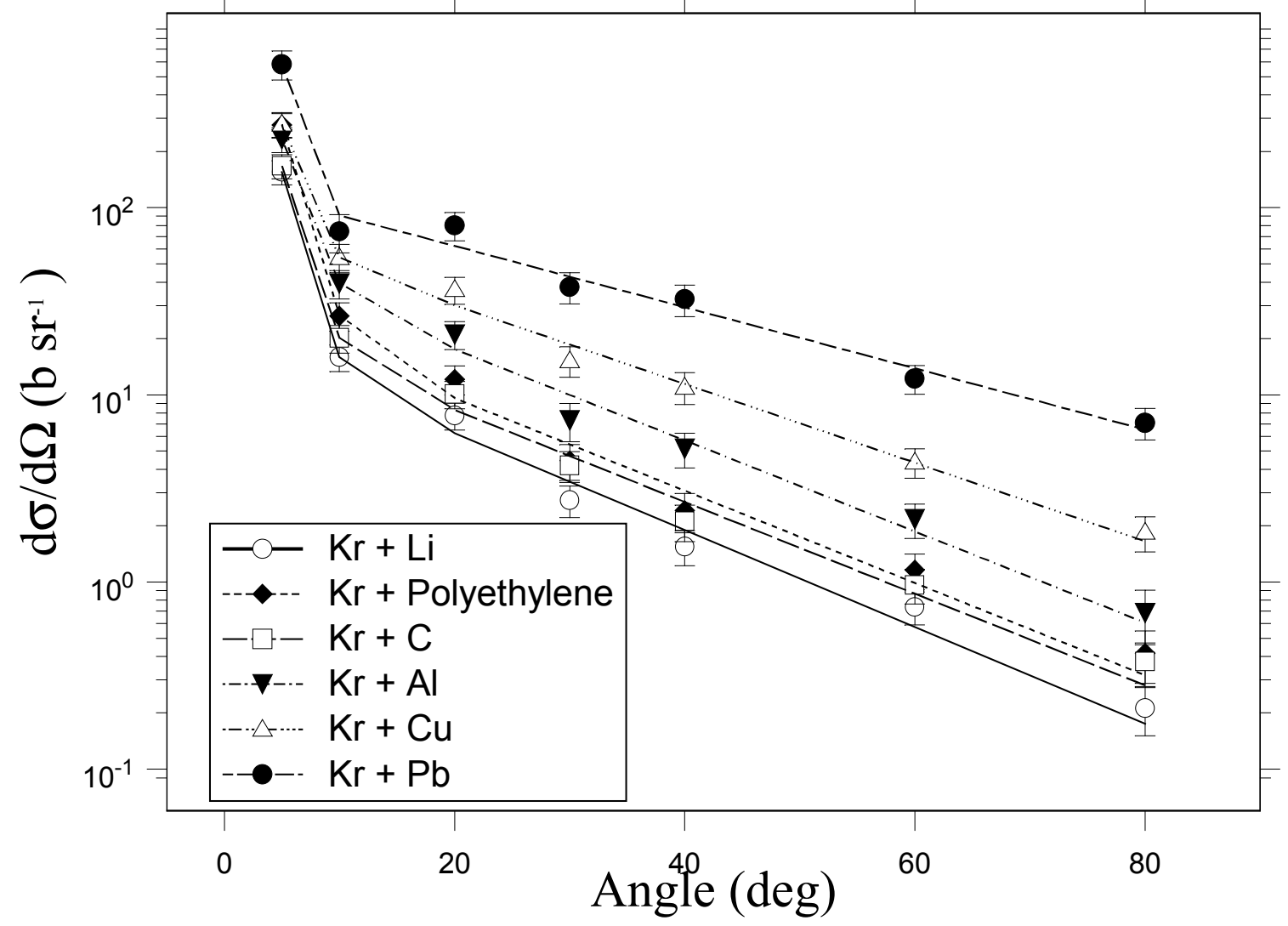

Fig. 27. Angular distribution from the $400 \mathrm{MeV} /$ nucleon $\mathrm{Kr}$ systems for $\mathrm{E}_{\mathrm{n}}>10$ MeV. The lines show a fit to the data using Eqn. 5. 


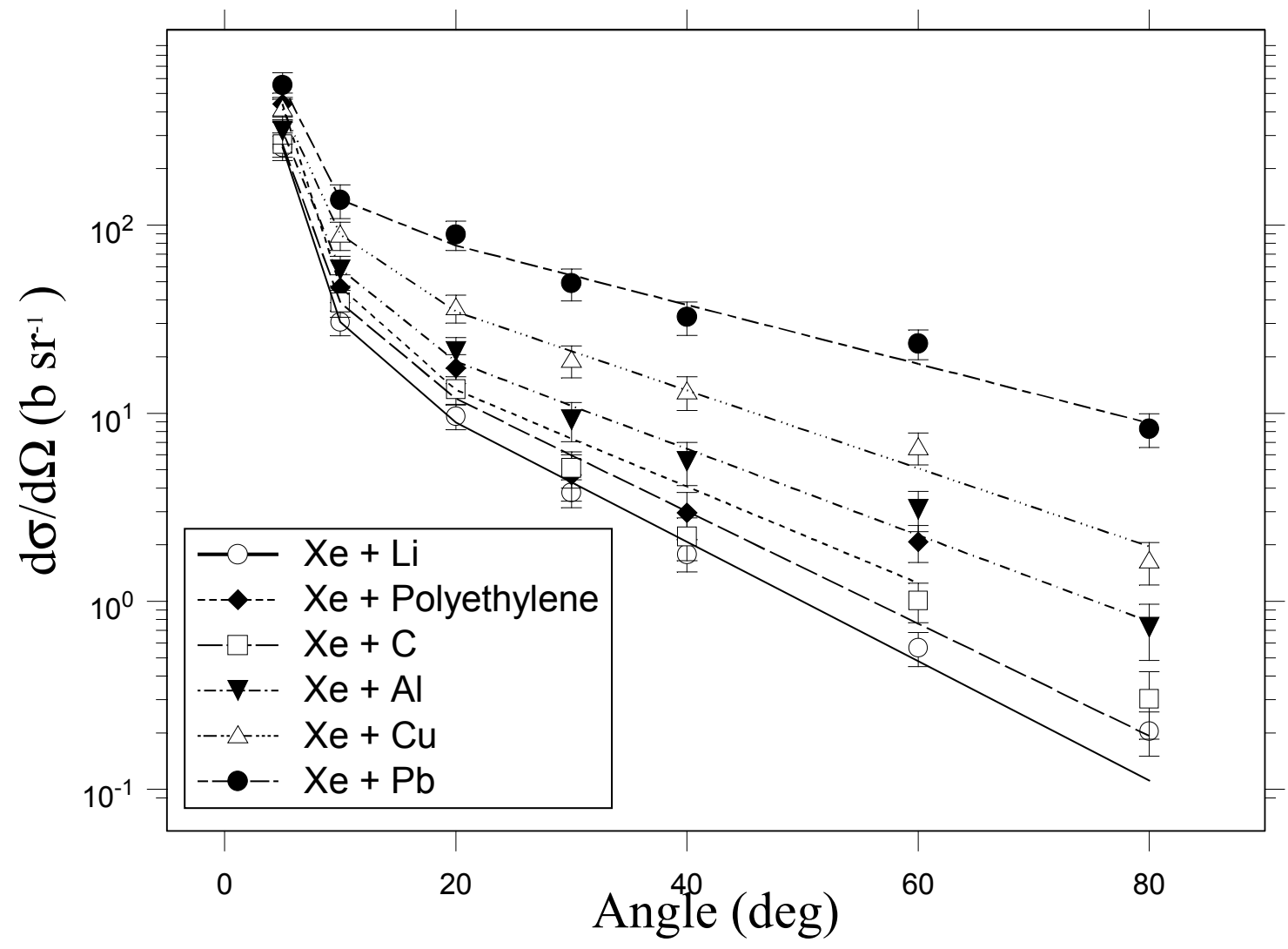

Fig. 28. Angular distribution from the $400 \mathrm{MeV} /$ nucleon Xe systems for $E_{n}>10$ MeV. The lines show a fit to the data using Eqn. 5. 


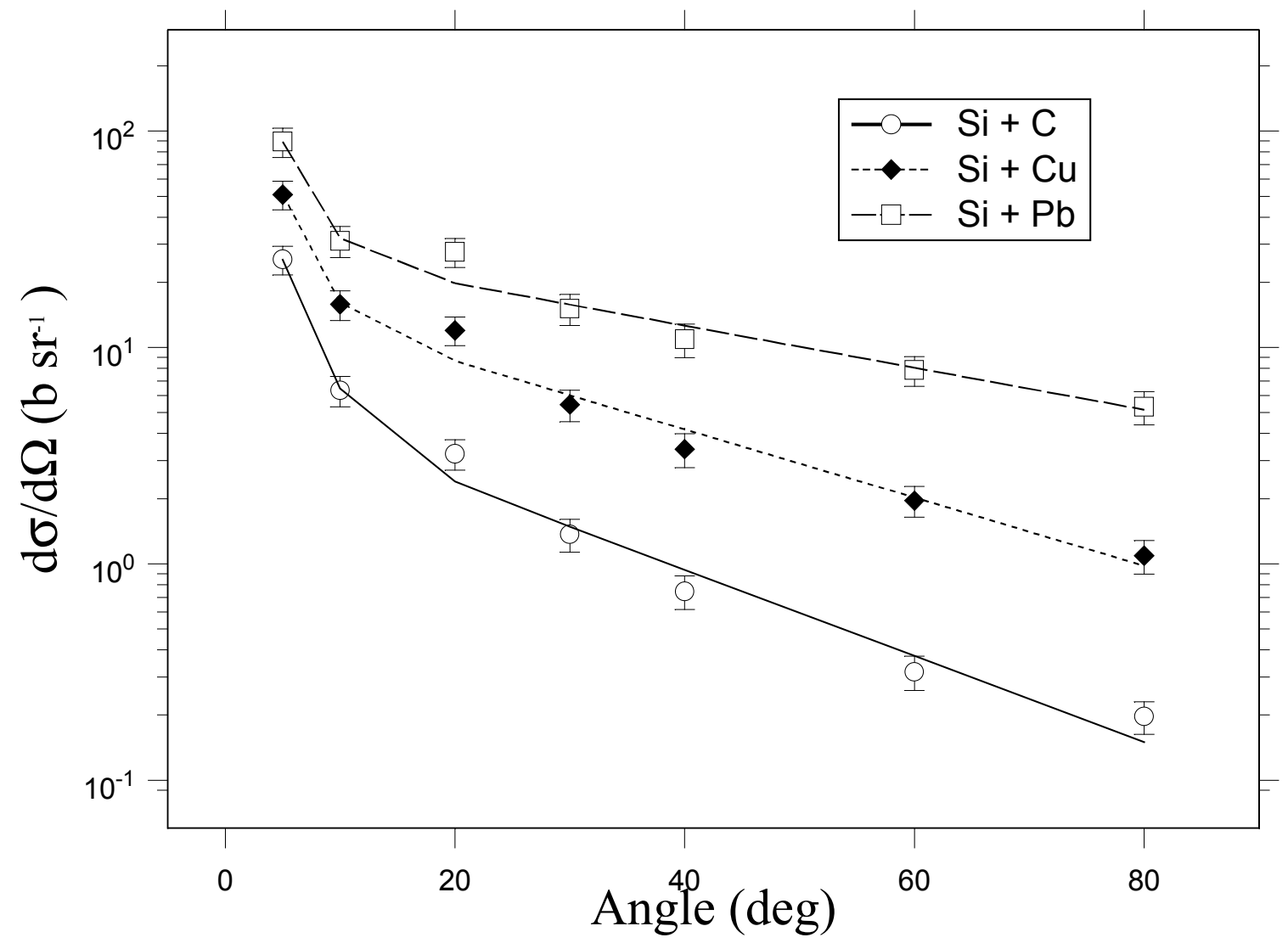

Fig. 29. Angular distribution from the $600 \mathrm{MeV} /$ nucleon Si systems for $E_{n}>10$ MeV. The lines show a fit to the data using Eqn. 5. 


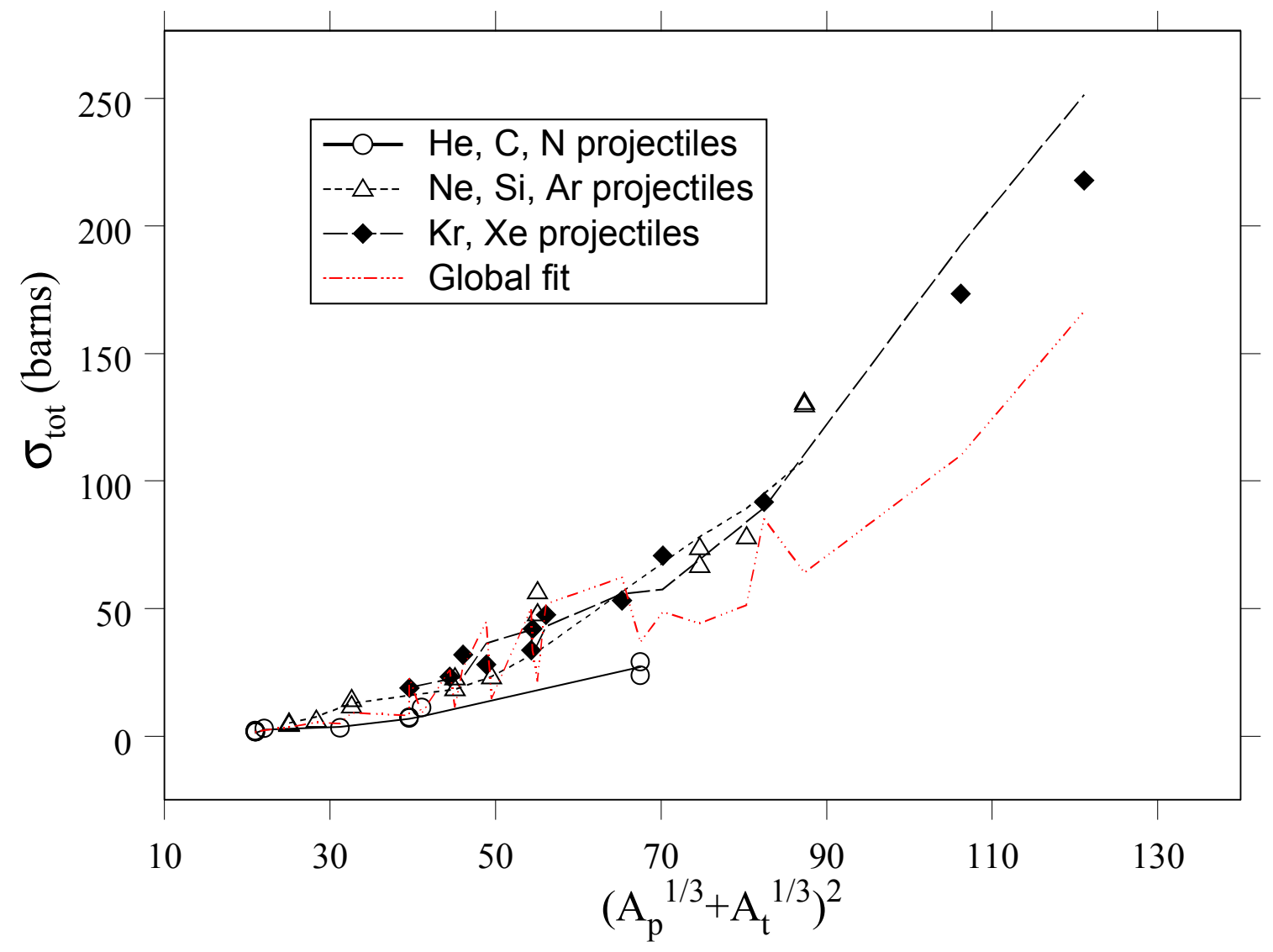

Fig. 30. Total cross sections for neutrons above $10 \mathrm{MeV}$ and angles between 0 and 180 degrees, as a function of a parameter that is proportional to the geometric cross sections. The lines indicate fits to the data using Eqn. 6. 


\section{Table I}

Target species and thickness $\left(\mathrm{g} / \mathrm{cm}^{2}\right)$ used with the indicated beams

\begin{tabular}{|c|c|c|}
\hline $\begin{array}{l}\text { Beam (energy) } \\
\text { (MeV/nucleon) }\end{array}$ & Target & $\begin{array}{l}\text { Thickness } \\
\left(\mathrm{g} / \mathrm{cm}^{2}\right)\end{array}$ \\
\hline He (230) & $\begin{array}{l}\mathrm{Al} \\
\mathrm{Cu}\end{array}$ & $\begin{array}{l}5.40(2.0 \mathrm{~cm}) \\
5.38(0.6 \mathrm{~cm})\end{array}$ \\
\hline $\mathrm{N}(400)$ & $\begin{array}{l}\mathrm{C} \\
\mathrm{Cu}\end{array}$ & $\begin{array}{l}1.78(1.0 \mathrm{~cm}) \\
2.69(0.3 \mathrm{~cm})\end{array}$ \\
\hline $\mathrm{Si}(600)$ & $\begin{array}{l}\mathrm{C} \\
\mathrm{Cu} \\
\mathrm{Pb} \\
\end{array}$ & $\begin{array}{l}1.80(1.0 \mathrm{~cm}) \\
3.58(0.4 \mathrm{~cm}) \\
4.54(0.4 \mathrm{~cm}) \\
\end{array}$ \\
\hline $\mathrm{Fe}(500)$ & $\begin{array}{l}\mathrm{Li} \\
\mathrm{CH}_{2} \\
\mathrm{Al} \\
\end{array}$ & $\begin{array}{l}0.903(1.7 \mathrm{~cm}) \\
0.957(1.05 \mathrm{~cm}) \\
1.285(0.476 \mathrm{~cm})\end{array}$ \\
\hline $\mathrm{Kr}(400)$ & $\begin{array}{l}\mathrm{Li} \\
\mathrm{C} \\
\mathrm{CH}_{2} \\
\mathrm{Al} \\
\mathrm{Cu} \\
\mathrm{Pb} \\
\end{array}$ & $\begin{array}{l}0.47(0.885 \mathrm{~cm}) \\
0.55(0.3 \mathrm{~cm}) \\
0.46(0.5 \mathrm{~cm}) \\
0.54(0.2 \mathrm{~cm}) \\
0.90(0.1 \mathrm{~cm}) \\
1.02(0.09 \mathrm{~cm}) \\
\end{array}$ \\
\hline $\mathrm{Xe}(400)$ & $\begin{array}{l}\mathrm{Li} \\
\mathrm{C} \\
\mathrm{CH}_{2} \\
\mathrm{Al} \\
\mathrm{Cu} \\
\mathrm{Pb}\end{array}$ & $\begin{array}{l}0.48(0.9 \mathrm{~cm}) \\
0.27(0.15 \mathrm{~cm}) \\
0.20(0.22 \mathrm{~cm}) \\
0.26(0.095 \mathrm{~cm}) \\
0.45(0.05 \mathrm{~cm}) \\
0.57(0.05 \mathrm{~cm})\end{array}$ \\
\hline
\end{tabular}


Table II

Neutron detector information. The uncertainty in the solid angle is reported as a percentage.

\begin{tabular}{|c|c|c|c|}
\hline Detector & $\begin{array}{c}\text { Flight Path Length } \\
(\mathrm{cm})\end{array}$ & $\begin{array}{c}\text { Lab angle } \\
(\mathrm{deg})\end{array}$ & $\begin{array}{c}\text { Solid angle } \\
(\mathrm{msr})\end{array}$ \\
\hline \hline N1 & 506 & 5 & $0.494 \pm 5.0 \%$ \\
N2 & 506 & 10 & $0.494 \pm 5.0 \%$ \\
N3 & 456 & 20 & $0.608 \pm 5.6 \%$ \\
N4 & 456 & 30 & $0.608 \pm 5.6 \%$ \\
N5 & 406 & 40 & $0.767 \pm 6.2 \%$ \\
N6 & 356 & 60 & $0.998 \pm 7.1 \%$ \\
N7 & 306 & 80 & $1.35 \pm 8.3 \%$ \\
\hline
\end{tabular}

Table III

Systematic uncertainties as a function of detector number, expressed as a percentage.

Adding the individual uncertainties in quadrature yields the total systematic uncertainty listed in column 7.

\begin{tabular}{|l|l|l|l|l|l|l|}
\hline $\begin{array}{l}\text { Detector } \\
\text { number }\end{array}$ & $\begin{array}{l}\text { Detection } \\
\text { Efficiency }\end{array}$ & $\begin{array}{l}\text { Flux } \\
\text { Attenuation }\end{array}$ & $\begin{array}{l}\text { Solid } \\
\text { Angle }\end{array}$ & $\begin{array}{l}\text { QDC } \\
\text { calibration }\end{array}$ & $\begin{array}{l}\text { Beam } \\
\text { Integration }\end{array}$ & Total \\
\hline \hline N1 & 10 & 7 & 5.0 & 5.2 & 4 & 14.7 \\
N2 & 10 & 7 & 5.0 & 5.4 & 4 & 14.8 \\
N3 & 10 & 7 & 5.6 & 3.8 & 4 & 14.5 \\
N4 & 10 & 7 & 5.6 & 6.3 & 4 & 15.4 \\
N5 & 10 & 7 & 6.2 & 9.1 & 4 & 16.9 \\
N6 & 10 & 7 & 7.1 & 3.8 & 4 & 15.2 \\
N7 & 10 & 7 & 8.3 & 7.9 & 4 & 17.2 \\
\hline
\end{tabular}


Table IV

Parameters from moving source fits to the indicated systems. The first part of the table contains the parameters of the projectile fragmentation source, and the second part contains the parameters from the two Maxwellian sources (see Eqns. 1 and 4).

\begin{tabular}{|l|l|c|c|c|}
\hline Beam & Target & \multicolumn{3}{|c|}{ Projectile fragmentation parameters } \\
\hline$(\mathrm{AMeV})$ & & $\mathrm{N}_{1}$ & $\sigma(\mathrm{MeV} / \mathrm{c})$ & $\beta$ \\
\hline $230 \mathrm{He}$ & $\mathrm{Al}$ & $(1.67 \pm 0.18) \times 10^{-8}$ & $75.9 \pm 4.0$ & $0.559 \pm 0.004$ \\
$230 \mathrm{He}$ & $\mathrm{Cu}$ & $(5.1 \pm 0.6) \times 10^{-8}$ & $68.8 \pm 3.0$ & $0.553 \pm 0.004$ \\
$400 \mathrm{~N}$ & $\mathrm{C}$ & $(8.7 \pm 0.8) \times 10^{-8}$ & $67.6 \pm 2.4$ & $0.696 \pm 0.002$ \\
$400 \mathrm{~N}$ & $\mathrm{Cu}$ & $(1.22 \pm 0.17) \times 10^{-7}$ & $78.2 \pm 4.1$ & $0.690 \pm 0.004$ \\
$400 \mathrm{Kr}$ & $\mathrm{Li}$ & $(1.61 \pm 0.04) \times 10^{-6}$ & $71.6 \pm 0.6$ & $0.724 \pm 0.001$ \\
$400 \mathrm{Kr}$ & $\mathrm{C}$ & $(1.66 \pm 0.06) \times 10^{-6}$ & $72.9 \pm 0.8$ & $0.721 \pm 0.001$ \\
$400 \mathrm{Kr}$ & $\mathrm{CH} 2$ & $(3.23 \pm 0.10) \times 10^{-6}$ & $69.0 \pm 0.7$ & $0.719 \pm 0.001$ \\
$400 \mathrm{Kr}$ & $\mathrm{Al}$ & $(2.31 \pm 0.15) \times 10^{-6}$ & $72.7 \pm 1.4$ & $0.712 \pm 0.001$ \\
$400 \mathrm{Kr}$ & $\mathrm{Cu}$ & $(3.23 \pm 0.28) \times 10^{-6}$ & $68.9 \pm 1.9$ & $0.702 \pm 0.002$ \\
$400 \mathrm{Kr}$ & $\mathrm{Pb}$ & $(1.63 \pm 0.79) \times 10^{-5}$ & $44.5 \pm 6$ & $0.694 \pm 0.003$ \\
$400 \mathrm{Xe}$ & $\mathrm{Li}$ & $(4.43 \pm 0.08) \times 10^{-6}$ & $60.8 \pm 0.4$ & $0.6957 \pm 0.0003$ \\
$400 \mathrm{Xe}$ & $\mathrm{C}$ & $(4.58 \pm 0.18) \times 10^{-6}$ & $61.2 \pm 0.8$ & $0.6915 \pm 0.0007$ \\
$400 \mathrm{Xe}$ & $\mathrm{CH} 2$ & $(7.30 \pm 0.27) \times 10^{-6}$ & $62.1 \pm 0.8$ & $0.698 \pm 0.001$ \\
$400 \mathrm{Xe}$ & $\mathrm{Al}$ & $(4.37 \pm 0.32) \times 10^{-6}$ & $66.4 \pm 1.8$ & $0.690 \pm 0.0013$ \\
$400 \mathrm{Xe}$ & $\mathrm{Cu}$ & $(6.27 \pm 0.52) \times 10^{-6}$ & $64.1 \pm 1.5$ & $0.682 \pm 0.0015$ \\
$400 \mathrm{Xe}$ & $\mathrm{Pb}$ & $(9.54 \pm 1.88) \times 10^{-6}$ & $62.6 \pm 4.0$ & $0.676 \pm 0.002$ \\
$500 \mathrm{Fe}$ & $\mathrm{Li}$ & $(2.25 \pm 0.77) \times 10^{-8}$ & $203 \pm 17$ & $0.716 \pm 0.023$ \\
$500 \mathrm{Fe}$ & $\mathrm{Al}$ & $(8.1 \pm 44) \times 10^{-6}$ & $101 \pm 101$ & $0.74 \pm 0.10$ \\
$600 \mathrm{Si}$ & $\mathrm{C}$ & $(1.31 \pm 0.06) \times 10^{-7}$ & $91.3 \pm 1.4$ & $0.768 \pm 0.001$ \\
$600 \mathrm{Si}$ & $\mathrm{Cu}$ & $(1.67 \pm 0.11) \times 10^{-7}$ & $106 \pm 3$ & $0.763 \pm 0.002$ \\
$600 \mathrm{Si}$ & $\mathrm{Pb}$ & $(2.45 \pm 0.31) \times 10^{-7}$ & $116 \pm 6$ & $0.751 \pm 0.003$ \\
\hline
\end{tabular}

\begin{tabular}{|l|l|l|l|l|l|l|l|}
\hline Beam & Tgt & \multicolumn{3}{|c|}{ Pre-equilibrium source } & \multicolumn{3}{c|}{ Equilibrium Source } \\
\hline$(\mathrm{AMeV})$ & & \multicolumn{1}{|c|}{$\mathrm{N}$} & \multicolumn{1}{|c|}{$\tau(\mathrm{MeV})$} & \multicolumn{1}{c|}{$\beta$} & \multicolumn{1}{c|}{$\mathrm{N}$} & \multicolumn{1}{c|}{$\tau(\mathrm{MeV})$} & \multicolumn{1}{c|}{$\beta$} \\
\hline \hline $230 \mathrm{He}$ & $\mathrm{Al}$ & $0.88 \pm 0.05$ & $44.7 \pm 1.8$ & $0.311 \pm 0.013$ & $0.87 \pm 0.09$ & $14.8 \pm 2.0$ & $0.00 \pm 0.13$ \\
$230 \mathrm{He}$ & $\mathrm{Cu}$ & $2.19 \pm 0.14$ & $49.0 \pm 1.9$ & $0.250 \pm 0.014$ & $2.14 \pm 0.30$ & $7.9 \pm 1.0$ & $0.027 \pm 0.012$ \\
$400 \mathrm{~N}$ & $\mathrm{C}$ & $1.83 \pm 0.09$ & $62.8 \pm 2.5$ & $0.516 \pm 0.010$ & $1.24 \pm 0.17$ & $21.7 \pm 2.3$ & $0.007 \pm 0.024$ \\
$400 \mathrm{~N}$ & $\mathrm{Cu}$ & $7.2 \pm 0.3$ & $73.0 \pm 2.4$ & $0.408 \pm 0.013$ & $4.7 \pm 0.4$ & $13.0 \pm 1.1$ & $0.033 \pm 0.012$ \\
$400 \mathrm{Kr}$ & $\mathrm{Li}$ & $8.8 \pm 0.4$ & $76 \pm 4$ & $0.419 \pm 0.020$ & $1.2 \pm 0.5$ & $10.4 \pm 8.8$ & $0.044 \pm 0.068$ \\
$400 \mathrm{Kr}$ & $\mathrm{C}$ & $9.7 \pm 0.8$ & $82.7 \pm 5.5$ & $0.418 \pm 0.020$ & $2.1 \pm 1.8$ & $12.9 \pm 11.5$ & $0.079 \pm 0.133$ \\
$400 \mathrm{Kr}$ & $\mathrm{CH}_{2}$ & $14.1 \pm 0.8$ & $84 \pm 7$ & $0.43 \pm 0.02$ & $1.4 \pm 0.8$ & $14 \pm 12$ & $0.104 \pm 0.08$ \\
$400 \mathrm{Kr}$ & $\mathrm{Al}$ & $22.3 \pm 1.0$ & $101 \pm 8$ & $0.438 \pm 0.019$ & $4.7 \pm 1.3$ & $9.4 \pm 3.8$ & $0.098 \pm 0.071$
\end{tabular}




\begin{tabular}{|l|l|l|l|l|l|l|l|}
$400 \mathrm{Kr}$ & $\mathrm{Cu}$ & $34.2 \pm 1.5$ & $107 \pm 12$ & $0.44 \pm 0.02$ & $14.8 \pm 1.7$ & $36.7 \pm 7.4$ & $0.23 \pm 0.05$ \\
$400 \mathrm{Kr}$ & $\mathrm{Pb}$ & $129 \pm 5$ & $69 \pm 3$ & $0.327 \pm 0.015$ & $72 \pm 7$ & $8.0 \pm 1.1$ & $0.0 \pm 0.02$ \\
$400 \mathrm{Xe}$ & $\mathrm{Li}$ & $10.3 \pm 0.3$ & $48.6 \pm 1.5$ & $0.610 \pm 0.004$ & $2.4 \pm 0.4$ & $12.6 \pm 1.6$ & $0.0085 \pm 0.015$ \\
$400 \mathrm{Xe}$ & $\mathrm{C}$ & $12.0 \pm 0.8$ & $52 \pm 2$ & $0.600 \pm 0.011$ & $4.3 \pm 0.8$ & $70 \pm 6$ & $0.18 \pm 0.08$ \\
$400 \mathrm{Xe}$ & $\mathrm{CH}_{2}$ & $14.0 \pm 0.9$ & $77 \pm 13$ & $0.573 \pm 0.085$ & $18 \pm 3$ & $70 \pm 18$ & $0.0 \pm 0.063$ \\
$400 \mathrm{Xe}$ & $\mathrm{Al}$ & $25.1 \pm 1.8$ & $70 \pm 5$ & $0.538 \pm 0.017$ & $40 \pm 37$ & $2.9 \pm 0.4$ & $0.132 \pm 0.015$ \\
$400 \mathrm{Xe}$ & $\mathrm{Cu}$ & $48 \pm 3$ & $74 \pm 4$ & $0.514 \pm 0.014$ & $17.3 \pm 3.2$ & $23 \pm 5$ & $0.074 \pm 0.035$ \\
$400 \mathrm{Xe}$ & $\mathrm{Pb}$ & $142 \pm 9$ & $78 \pm 3$ & $0.396 \pm 0.019$ & $67 \pm 10$ & $14.9 \pm 2.7$ & $0.044 \pm 0.025$ \\
$500 \mathrm{Fe}$ & $\mathrm{Li}$ & $4.5 \pm 0.5$ & $83 \pm 6$ & $0.47 \pm 0.03$ & $3.0 \pm 0.5$ & $14 \pm 4$ & $0.022 \pm 0.046$ \\
$500 \mathrm{Fe}$ & $\mathrm{Al}$ & $8.0 \pm 0.9$ & $85 \pm 10$ & $0.62 \pm 0.08$ & $10.5 \pm 1.0$ & $73 \pm 8$ & $0.186 \pm 0.103$ \\
$600 \mathrm{Si}$ & $\mathrm{C}$ & $3.57 \pm 0.14$ & $83 \pm 6$ & $0.54 \pm 0.03$ & $2.8 \pm 0.2$ & $33 \pm 4$ & $0.0 \pm 0.017$ \\
$600 \mathrm{Si}$ & $\mathrm{Cu}$ & $14.3 \pm 0.4$ & $90 \pm 4$ & $0.41 \pm 0.02$ & $14.3 \pm 0.5$ & $19.9 \pm 1.5$ & $0.0 \pm 0.008$ \\
$600 \mathrm{Si}$ & $\mathrm{Pb}$ & $55.2 \pm 1.5$ & $100 \pm 4$ & $0.213 \pm 0.021$ & $69.0 \pm 1.5$ & $12.7 \pm 0.6$ & $0.0 \pm 0.018$ \\
\hline
\end{tabular}

Table V

Parameters from fits to the indicated angular distributions using Eqn. 5.

\begin{tabular}{|c|c|c|c|c|c|}
\hline $\begin{array}{l}\text { Beam } \\
(\mathrm{AMeV})\end{array}$ & Target & $\begin{array}{c}\mathrm{a}_{1} \\
(\mathrm{~b} / \mathrm{sr})\end{array}$ & $\begin{array}{c}\mathrm{a}_{2} \\
\text { (1/radian) }\end{array}$ & $\begin{array}{c}\mathrm{a}_{3} \\
(\mathrm{~b} / \mathrm{sr})\end{array}$ & $\begin{array}{c}\mathrm{a}_{4} \\
\text { (1/radian) }\end{array}$ \\
\hline $230 \mathrm{He}$ & $\mathrm{Al}$ & $1.76 \pm 0.16$ & $4.4 \pm 0.8$ & $0.225 \pm 0.18$ & $0.72 \pm 0.57$ \\
\hline $230 \mathrm{He}$ & $\mathrm{Cu}$ & $3.72 \pm 0.06$ & $5.96 \pm 0.08$ & $0.88 \pm 0.01$ & $1.11 \pm 0.01$ \\
\hline $400 \mathrm{~N}$ & $\mathrm{C}$ & $190 \pm 150$ & $42 \pm 22$ & $3.4 \pm 0.3$ & $2.63 \pm 0.13$ \\
\hline $400 \mathrm{~N}$ & $\mathrm{Cu}$ & $34 \pm 24$ & $18 \pm 8$ & $8.1 \pm 0.8$ & $1.92 \pm 0.11$ \\
\hline $400 \mathrm{Kr}$ & $\mathrm{Li}$ & $4080 \pm 1420$ & $39.3 \pm 4.1$ & $20.1 \pm 2.8$ & $3.49 \pm 0.22$ \\
\hline $400 \mathrm{Kr}$ & $\mathrm{C}$ & $3940 \pm 1570$ & $37.6 \pm 4.7$ & $25.7 \pm 3.9$ & $3.24 \pm 0.23$ \\
\hline $400 \mathrm{Kr}$ & $\mathrm{CH}_{2}$ & $6750 \pm 2220$ & $37.6 \pm 3.9$ & $29.8 \pm 5.6$ & $3.25 \pm 0.28$ \\
\hline $400 \mathrm{Kr}$ & Al & $4190 \pm 2350$ & $35.4 \pm 6.6$ & $53.6 \pm 8.7$ & $3.21 \pm 0.24$ \\
\hline $400 \mathrm{Kr}$ & $\mathrm{Cu}$ & $8630 \pm 5530$ & $42 \pm 13$ & $79 \pm 8$ & $2.77 \pm 0.14$ \\
\hline $400 \mathrm{Kr}$ & $\mathrm{Pb}$ & $(9.6 \pm 5.1) \times 10^{6}$ & $114 \pm 15$ & $132 \pm 13$ & $2.16 \pm 0.10$ \\
\hline $400 \mathrm{Xe}$ & $\mathrm{Li}$ & $4230 \pm 1440$ & $39 \pm 4$ & $20.5 \pm 3$ & $3.42 \pm 0.23$ \\
\hline $400 \mathrm{Xe}$ & $\mathrm{C}$ & $3680 \pm 900$ & $31.4 \pm 3.0$ & $47 \pm 9$ & $3.94 \pm 0.34$ \\
\hline $400 \mathrm{Xe}$ & $\mathrm{CH}_{2}$ & $7260 \pm 1900$ & $33 \pm 3$ & $43 \pm 13$ & $3.38 \pm 0.52$ \\
\hline $400 \mathrm{Xe}$ & Al & $2840 \pm 780$ & $26.7 \pm 3.2$ & $53.4 \pm 9.8$ & $3.03 \pm 0.26$ \\
\hline $400 \mathrm{Xe}$ & $\mathrm{Cu}$ & $3560 \pm 1000$ & $26.8 \pm 3.3$ & $90 \pm 11$ & $2.74 \pm 0.16$ \\
\hline $400 \mathrm{Xe}$ & $\mathrm{Pb}$ & $7160 \pm 6300$ & $32.4 \pm 10.2$ & $160 \pm 19$ & $2.07 \pm 0.13$ \\
\hline $600 \mathrm{Si}$ & $\mathrm{C}$ & $156 \pm 31$ & $23.1 \pm 2.3$ & $5.9 \pm 0.7$ & $2.63 \pm 0.14$ \\
\hline $600 \mathrm{Si}$ & $\mathrm{Cu}$ & $354 \pm 117$ & $26.2 \pm 3.8$ & $17.8 \pm 1.2$ & $2.08 \pm 0.07$ \\
\hline $600 \mathrm{Si}$ & $\mathrm{Pb}$ & $520 \pm 210$ & $24.5 \pm 4.5$ & $30.8 \pm 1.7$ & $1.28 \pm 0.05$ \\
\hline
\end{tabular}




\section{Table VI}

Total cross sections for the indicated systems. Columns $3-5$ show the integrated cross sections from 0 to 90 degrees. Column six shows the deduced total cross sections from 0 to 180 degrees.

\begin{tabular}{|l|l|l|l|l|l|}
\hline $\begin{array}{c}\text { Beam } \\
(\mathrm{AMeV})\end{array}$ & Target & $\begin{array}{c}>5 \mathrm{MeV}^{(\mathrm{w} / \mathrm{c})} \\
\text { (barns) }\end{array}$ & $\begin{array}{c}>10 \mathrm{MeV}^{(\mathrm{w} / \mathrm{c})} \\
(\text { barns })\end{array}$ & $\begin{array}{c}>10 \mathrm{MeV}^{(\mathrm{w} / \mathrm{oc})} \\
(\text { barns })\end{array}$ & $\begin{array}{c}>10 \mathrm{MeV}^{(0-180)} \\
\text { (barns) }\end{array}$ \\
\hline $230 \mathrm{He}$ & $\mathrm{Al}$ & $1.39 \pm 0.10$ & $1.28 \pm 0.09$ & $1.06 \pm 0.07$ & 1.65 \\
$230 \mathrm{He}$ & $\mathrm{Cu}$ & $3.14 \pm 0.20$ & $2.68 \pm 0.17$ & $2.29 \pm 0.14$ & 3.20 \\
$400 \mathrm{~N}$ & $\mathrm{C}$ & $3.00 \pm 0.20$ & $2.92 \pm 0.20$ & $2.70 \pm 0.18$ & 2.96 \\
$400 \mathrm{~N}$ & $\mathrm{Cu}$ & $11.5 \pm 0.8$ & $10.6 \pm 0.7$ & $9.7 \pm 0.7$ & 11.2 \\
$400 \mathrm{Kr}$ & $\mathrm{Li}$ & no data & $18.8 \pm 1.5$ & $17.8 \pm 1.4$ & 18.85 \\
$400 \mathrm{Kr}$ & $\mathrm{C}$ & no data & $23.2 \pm 1.8$ & $22.4 \pm 1.7$ & 23.29 \\
$400 \mathrm{Kr}$ & $\mathrm{CH} 2$ & no data & $31.8 \pm 2.6$ & $30.7 \pm 2.5$ & 31.90 \\
$400 \mathrm{Kr}$ & $\mathrm{Al}$ & $42.4 \pm 3.2$ & $41.9 \pm 3.1$ & $40.4 \pm 3.0$ & 42.10 \\
$400 \mathrm{Kr}$ & $\mathrm{Cu}$ & $71.2 \pm 4.9$ & $70.0 \pm 4.8$ & $66.3 \pm 4.5$ & 70.76 \\
$400 \mathrm{Kr}$ & $\mathrm{Pb}$ & $179 \pm 13$ & $168 \pm 12$ & $160 \pm 12$ & 173.34 \\
$400 \mathrm{Xe}$ & $\mathrm{Li}$ & no data & $28.0 \pm 2.3$ & $26.5 \pm 2.2$ & 28.02 \\
$400 \mathrm{Xe}$ & $\mathrm{C}$ & no data & $33.6 \pm 2.7$ & $32.6 \pm 2.6$ & 33.64 \\
$400 \mathrm{Xe}$ & $\mathrm{CH} 2$ & no data & $47.4 * \pm 4.2$ & $46.0 * \pm 4.0$ & 47.51 \\
$400 \mathrm{Xe}$ & $\mathrm{Al}$ & no data & $52.9 \pm 4.2$ & $51.2 \pm 4.1$ & 53.19 \\
$400 \mathrm{Xe}$ & $\mathrm{Cu}$ & no data & $90.8 \pm 6.5$ & $87.5 \pm 6.3$ & 91.73 \\
$400 \mathrm{Xe}$ & $\mathrm{Pb}$ & no data & $210 \pm 15$ & $201 \pm 15$ & 217.89 \\
$600 \mathrm{Si}$ & $\mathrm{C}$ & no data & $6.20 \pm 0.40$ & $5.76 \pm 0.37$ & 6.28 \\
$600 \mathrm{Si}$ & $\mathrm{Cu}$ & $24.2 \pm 1.5$ & $22.5 \pm 1.4$ & $20.4 \pm 1.3$ & 23.36 \\
$600 \mathrm{Si}$ & $\mathrm{Pb}$ & $75.7 \pm 5.1$ & $66.7 \pm 4.4$ & $59.9 \pm 4.0$ & 78.21 \\
\hline
\end{tabular}

$(\mathrm{w} / \mathrm{c})$ - includes correction for attenuation of neutron flux (w/o c) - not corrected for attenuation of neutron flux * - integrated from 0 to 70 degrees 


\section{Table VII}

Fit parameters to the total cross sections $\left(0^{\circ}\right.$ to $\left.180^{\circ}\right)$ using Eqn. 6 .

\begin{tabular}{|l|l|l|l|c|}
\hline & \multicolumn{1}{|c|}{$\mathrm{C}$} & \multicolumn{1}{c|}{$\mathrm{f}_{\mathrm{p}}$} & $\mathrm{f}_{\mathrm{t}}$ & $\chi^{2}$ (d.o.f.) \\
\hline \hline $\mathrm{He}, \mathrm{C}$, N projectiles & $0.014 \pm 0.004$ & $1.0 \pm 0.5$ & $0.18 \pm 0.05$ & 0.61 \\
Ne, Si, Ar projectiles & $0.016 \pm 0.008$ & $1.0 \pm 0.5$ & $0.44 \pm 0.21$ & 0.65 \\
Kr, Xe projectiles & $0.014 \pm 0.006$ & $0.65 \pm 0.28$ & $0.78 \pm 0.31$ & 0.64 \\
All systems & $0.013 \pm 0.004$ & $0.90 \pm 0.22$ & $0.29 \pm 0.08$ & 1.89 \\
\hline
\end{tabular}

